The Astrophysical Journal, 627:432-445, 2005 July 1

(C) 2005. The American Astronomical Society. All rights reserved. Printed in U.S.A.

\title{
ABUNDANCE ANALYSES OF FIELD RV TAURI STARS. VI. AN EXTENDED SAMPLE
}

\author{
SUNETRA GIRIDHAR \\ Indian Institute of Astrophysics, II Block, Koramangala, Bangalore 560 034, India; giridhar@iiap.res.in \\ David L. LAmberT \\ McDonald Observatory, University of Texas, 1 University Station, Austin, TX 78712-1083; dll@astro.as.utexas.edu \\ BACHAM E. REDDY ${ }^{1}$ \\ Indian Institute of Astrophysics, II Block, Koramangala, Bangalore 560 034, India; ereddy@iiap.res.in \\ Guillermo GonZalez \\ Department of Physics and Astronomy, Iowa State University, Ames, IA 50011-3160; gonzog@iastate.edu \\ AND \\ DAVID YONG \\ Department of Astronomy, University of Texas, C-1400, Austin, TX 78712-1083; tofu@astro.as.utexas.edu \\ Received 2004 July 8; accepted 2005 March 13
}

\begin{abstract}
An abundance analysis is presented and discussed for a sample of 14 RV Tauri stars. The present abundance data and those from our previous papers and by other workers are combined in an attempt to further understanding of the dust-gas separation process that afflicts many RV Tauri variables. We propose that a star's intrinsic (i.e., initial) metallicity is given by the photospheric zinc abundance. Variables warmer than about $5000 \mathrm{~K}$ and with an initial metallicity $[\mathrm{Fe} / \mathrm{H}] \geq-1$ are affected by dust-gas separation. Variables of all metallicities and cooler than about $T_{\text {eff }} \simeq 5000 \mathrm{~K}$ are unaffected by dust-gas separation. The RV Tauri variables show a spread in their C abundances, with the lower boundary of the points in the $\mathrm{C}$ versus $\mathrm{Zn}$ plane falling close to the predicted trend for giants after the first dredge-up. The upper boundary is inhabited by a few stars that are carbon-rich. The $\mathrm{O}$ abundances in the mean follow the predicted trend from unevolved stars, in line with the expectation that photospheric $\mathrm{O}$ abundance is unaffected by the first dredge-up. An evolutionary scenario involving mass loss by a first-ascent or early-AGB red giant, the primary star of a binary, is sketched.
\end{abstract}

Subject headings: stars: abundances — stars: AGB and post-AGB — stars: variables: other

\section{INTRODUCTION}

In this series of papers, we have been exploring the chemical compositions of RV Tauri variables in the Galactic field. This exploration concludes here with abundance analyses reported for 14 variables. Of these, 12 are analyzed for the first time, one (LR Sco) was previously analyzed by us before its status as an RV Tauri variable was appreciated by Lloyd Evans (1999), and one (DY Ori) is analyzed more thoroughly than in our earlier attempt.

Beginning with the analysis of the southern RV Tauri variable IW Car (Giridhar et al. 1994 [Paper I]), we have shown that the atmospheric composition of an RV Tauri star may be abnormal in the sense that the photospheric abundance anomalies are roughly correlated with the predicted condensation temperature for lowpressure gas of solar composition. In particular, elements (e.g., $\mathrm{Al}, \mathrm{Ca}, \mathrm{Ti}$, and $\mathrm{Sc}$ ) with the highest condensation temperatures $(\sim 1600 \mathrm{~K})$ may be seriously underabundant relative to their abundance expected from the abundances of elements (e.g., S and $\mathrm{Zn}$ ) of low condensation temperature (Gonzalez et al. 1997a, hereafter Paper II; Gonzalez et al. 1997b, hereafter Paper III; Giridhar et al. 1998, hereafter Paper IV; Giridhar et al. 2000, hereafter Paper V). Our findings were confirmed by independent analyses of several RV Tauri stars by Van Winckel et al. (1998), Maas et al. (2002), and Maas (2003). These abundance anoma-

\footnotetext{
${ }^{1}$ Visiting Observer, Cerro Tololo Inter-American Observatory, which is operated by the Association of Universities for Research in Astronomy, Inc., under contract with the National Science Foundation.
}

lies imply that the RV Tauri's photosphere is deficient in those elements that condense most readily into dust grains. We refer to the principal operation necessary to achieve the deficiencies as dust-gas separation.

If such a separation is to affect the photospheric composition, three conditions must be met. First (condition A), a site for dust formation must exist near the star. Two proposed locations compete to meet this condition: the wind off the star or a circumstellar or circumbinary disk. Second (condition B), a mechanism must be identified to separate dust from gas. It has been supposed that radiation pressure on the dust grains drives them through the gas and away from the star(s). Third (condition C), the dust-free gas accreted by the star must become the dominant constituent of the star's photosphere. Here the principal issue is that the photosphere is a part of a convective envelope. In order for the photosphere to assume a composition dominated by dust-free gas, the mass of the envelope must be small relative to the mass of accreted gas.

Our earlier analyses have shown that the severity of the atmospheric abundance anomalies differs from one RV Tauri to another. These differences presumably are clues to the circumstances under which the above conditions are or are not met. Taking them in inverse order, the following may be noted. Relevant to condition C, the coolest RV Tauri variables independent of their metallicity are free of the abundance anomalies. One interpretation is that these stars have deep convective envelopes that dilute accreted gas and prevent the appearance of abundance anomalies even when gas, but not dust, is accreted (Paper V). Stars of intrinsically low metallicity, such as the variables in 
TABLE 1

The Program Stars

\begin{tabular}{|c|c|c|c|c|c|}
\hline Star & Date & $\begin{array}{l}\text { Radial Velocity } \\
\quad\left(\mathrm{km} \mathrm{s}^{-1}\right)\end{array}$ & $\begin{array}{l}\text { Period }^{\mathrm{a}} \\
\text { (days) }\end{array}$ & Spectroscopic Group ${ }^{\mathrm{b}}$ & Photometric Type ${ }^{\mathrm{a}}$ \\
\hline UY CMa ..................... & 1999 Nov 1 & +128 & 114.6 & B & $\mathrm{a}$ \\
\hline RX Cap ............................. & 1999 Nov 2 & -123 & 67.9 & A & $\ldots$ \\
\hline \multirow[t]{2}{*}{ 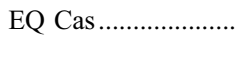 } & 1999 Aug 19 & -158 & 58.3 & $\mathrm{~B}$ ? & $\mathrm{a}$ \\
\hline & 2001 Aug 19 & -158 & 58.3 & $\mathrm{~B}$ ? & $\mathrm{a}$ \\
\hline DF Cyg ......................... & 1999 Nov 1 & -14 & 49.8 & A & $\ldots$ \\
\hline HP Lyr...................... & 2002 Nov 14 & -107 & $69.3^{c}$ & $\ldots$ & $\ldots$ \\
\hline 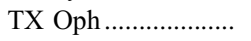 & 1999 Aug 19 & -166 & 135.0 & A & $\ldots$ \\
\hline \multirow[t]{2}{*}{ 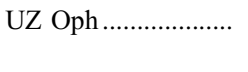 } & $2001 \mathrm{Jul} 13$ & -95 & 87.4 & A & $\mathrm{a}$ \\
\hline & 2002 Jun 30 & -90 & 87.4 & A & $\mathrm{a}$ \\
\hline DY Ori ............................ & 2002 Nov 14 & -8 & 83.4 & B & $\mathrm{a}$ \\
\hline AI Sco .......................... & 2002 Jun 30 & -35 & 71.0 & A & $\mathrm{b}$ \\
\hline LR Sco ........................ & 2002 Jun 22 & -18 & 104.4 & $\ldots$ & $\ldots$ \\
\hline AR Sgr.......................... & 1999 Oct 31 & -112 & 87.9 & $\mathrm{~A}$ ? & $\mathrm{a}$ \\
\hline 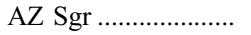 & 2002 Jun 19 & -110 & 113.6 & $\ldots$ & $\ldots$ \\
\hline BZ Sct.......................... & 1999 Jun 6 & +89 & 75.7 & $\ldots$ & $\ldots$ \\
\hline V Vul............................. & 1999 Aug 19 & -28 & 81.1 & A & $\mathrm{a}$ \\
\hline
\end{tabular}

${ }^{\text {a }}$ Data from Kukarkin et al. 1958 and Kholopov et al. 1992.

${ }^{b}$ Data from Preston et al. 1963.

c Data from Graczyk et al. 2002.

globular clusters (Gonzalez \& Lambert 1997; Russell 1998) and some high-velocity variables in the field, appear immune to the effects of a dust-gas separation. This fact is relevant to condition B: it is likely a consequence of the inability of radiation pressure on dust grains to force a separation of dust from gas when the mass fraction of dust is very low, as it is in a metal-poor cool environment. Accretion under these circumstances will not change the surface composition of the star even if it has a shallow envelope.

With respect to condition A, the extremely metal-deficient A-type post-AGB stars are evidence that dust-gas separation does not have to occur in a stellar wind (Van Winckel 2003). The prototypical example is HR $4049\left(T_{\text {eff }}=7600 \mathrm{~K}\right)$, which has an extensive infrared excess from dust, but its stellar wind, if it exists, is surely too hot to be the site of dust formation. Yet, dust-gas separation has provided a photospheric abundance of $[\mathrm{Fe} / \mathrm{H}] \simeq-4.8$ for a star with an initial abundance $[\mathrm{Fe} / \mathrm{H}] \approx$ -0.4. Van Winckel et al. (1995) showed that HR 4049 and other similar metal-deficient stars were spectroscopic binaries. The dust-gas separation is presumed to occur in a circumbinary dusty disk. Superposition of the pulsational velocity variation on an orbital variation complicates a demonstration that all RV Tauri stars affected by dust-gas separation are binaries. Certainly, several RV Tauri stars are known to be spectroscopic binaries. An assessment of the direct and indirect evidence (Van Winckel et al. 1998) led Van Winckel (2003) to write that "binarity may very well be a common phenomenon among RV Tauri stars." The question of dust-gas separation occurring in a wind off a cool star remains open.

An aim of this paper was to enlarge the sample of RV Tauri variables in order to test in more depth previous deductions about the dust-gas separation processes. We present abundances for the 14 variables listed in Table 1 . This new sample particularly increases the representation of high-velocity RV Tauri stars.

\section{OBSERVATIONS AND ABUNDANCE ANALYSES}

The program stars and dates of observation are listed in Table 1 along with the measured radial velocity, the pulsational period, the Preston spectroscopic type, A, B, or C (Preston et al. 1963), and the photometric type, $\mathrm{a}$ or $\mathrm{b}$. Photometric type $\mathrm{b}$ indicates that the light curve shows a long-term modulation.

A majority of the stars were observed with the McDonald Observatory $2.7 \mathrm{~m}$ Harlan J. Smith reflector with the CCDequipped 2Dcoudé spectrograph (Tull et al. 1995). A spectral resolving power $R=\lambda / \Delta \lambda \simeq 60,000$ was used, and a broad spectral range was covered in a single exposure. A signal-tonoise ratio $(\mathrm{S} / \mathrm{N})$ of $80-100$ over much of the spectral range was achieved. Figure 1 illustrates the quality of typical spectra.

The stars LR Sco and AZ Sgr were observed at the Cerro Tololo Inter-American Observatory (CTIO) in Chile with the

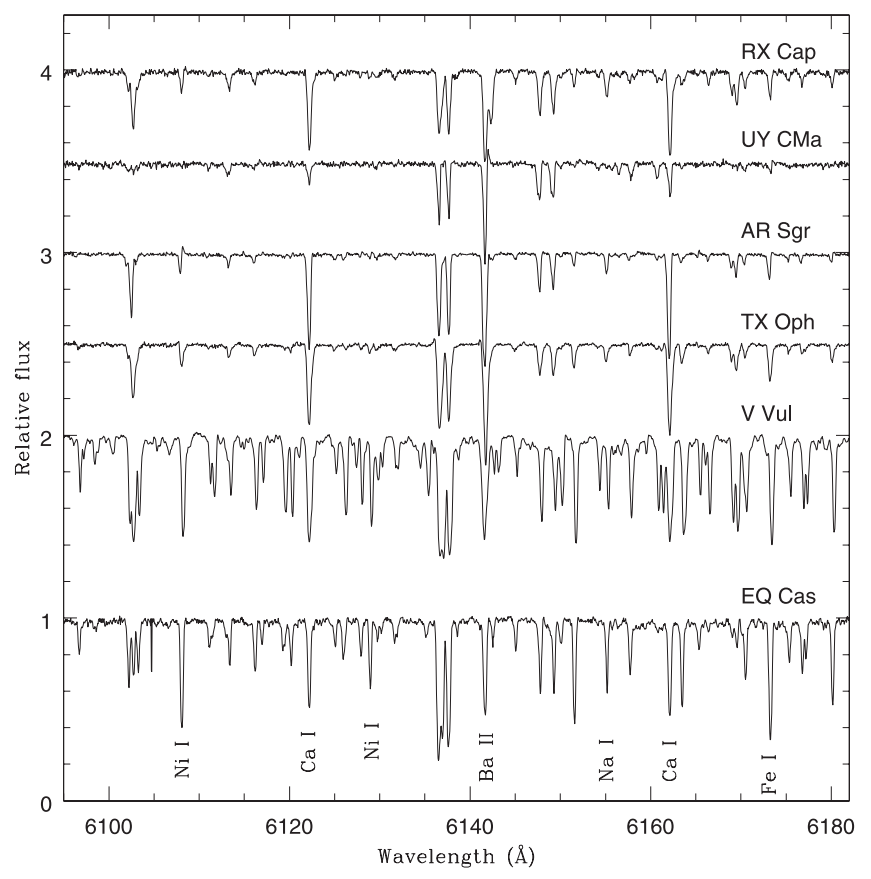

FIG. 1.-Spectra of a representative sample presented in descending order of temperature (top to bottom). The temperature of UY CMa is within $200 \mathrm{~K}$ of RX Cap and AR Sgr, but the lines of $\mathrm{Ca}$ I and $\mathrm{Fe}_{\mathrm{I}}$ are considerably weaker in UY CMa due to the dust-gas separation effect. 
TABLE 2

Stellar Parameters Derived from the Fe-Line Analyses

\begin{tabular}{|c|c|c|c|c|c|c|c|}
\hline \multirow[b]{2}{*}{$\mathrm{S}_{\mathrm{TAR}}$} & \multirow[b]{2}{*}{ UT Date } & \multirow[b]{2}{*}{ Model $T_{\text {eff }}, \log g,[\mathrm{Fe} / \mathrm{H}]^{\mathrm{a}}$} & \multirow{2}{*}{$\begin{array}{c}\xi_{t}^{\mathrm{b}} \\
\left(\mathrm{km} \mathrm{s}^{-1}\right)\end{array}$} & \multicolumn{2}{|c|}{$\mathrm{Fe} \mathrm{I}^{\mathrm{c}}$} & \multicolumn{2}{|c|}{$\mathrm{Fe}{ }_{I I}^{\mathrm{c}}$} \\
\hline & & & & $\log \epsilon$ & $n$ & $\log \epsilon$ & $n$ \\
\hline UY CMa .... & 1999 Nov 1 & $5500,0.0,-1.4$ & 5.0 & $6.15 \pm 0.17$ & 65 & $6.20 \pm 0.14$ & 11 \\
\hline RX Cap ....... & 1999 Nov 2 & $5800,1.0,-0.8$ & 4.8 & $6.69 \pm 0.16$ & 80 & $6.67 \pm 0.13$ & 16 \\
\hline \multirow[t]{2}{*}{ EQ Cas........ } & 1999 Aug 19 & $4500,0.0,-0.8$ & 4.6 & $6.68 \pm 0.13$ & 54 & $6.71 \pm 0.12$ & 13 \\
\hline & 2001 Aug 19 & $5300,0.7,-0.8$ & 3.6 & $6.73 \pm 0.15$ & 53 & $6.71 \pm 0.14$ & 9 \\
\hline DF Cyg ....... & 1999 Nov 1 & $4800,1.7,-0.0$ & 3.6 & $7.50 \pm 0.17$ & 22 & $7.36 \pm 0.18$ & 7 \\
\hline HP Lyr......... & 2002 Nov 14 & $6300,1.0,-1.0$ & 3.2 & $6.48 \pm 0.12$ & 99 & $6.52 \pm 0.10$ & 15 \\
\hline TX Oph....... & 1999 Aug 19 & $5000,0.5,-1.2$ & 4.9 & $6.24 \pm 0.17$ & 81 & $6.28 \pm 0.16$ & 17 \\
\hline UZ Oph...... & 2001 Jul 13 & $5000,0.5,-0.7$ & 3.6 & $6.78 \pm 0.14$ & 68 & $6.80 \pm 0.10$ & 6 \\
\hline UZ Oph...... & 2002 Jun 30 & $4800,0.0,-0.8$ & 3.6 & $6.70 \pm 0.15$ & 98 & $6.63 \pm 0.14$ & 14 \\
\hline DY Ori....... & 2002 Nov 14 & $6000,1.5,-2.0$ & 4.0 & $5.23 \pm 0.14$ & 30 & $5.27 \pm 0.12$ & 12 \\
\hline AI Sco ......... & 2002 Jun 30 & $5300,0.25,-0.7$ & 3.3 & $6.76 \pm 0.14$ & 38 & $6.82 \pm 0.10$ & 8 \\
\hline LR Sco ........ & 2002 Jun 22 & $6000,0.50,-0.2$ & 4.2 & $7.29 \pm 0.16$ & 50 & $7.24 \pm 0.11$ & 7 \\
\hline BZ Sct........ & 1999 Jun 6 & $6250,1.0,-0.8$ & 3.2 & $6.65 \pm 0.12$ & 63 & $6.64 \pm 0.13$ & 16 \\
\hline AR Sgr....... & 1999 Oct 31 & $5300,0.0,-1.5$ & 5.2 & $6.16 \pm 0.13$ & 62 & $6.09 \pm 0.14$ & 17 \\
\hline AZ Sgr ........ & 2002 Jun 19 & $4750,0.5,-1.6$ & 4.2 & $5.91 \pm 0.15$ & 45 & $5.96 \pm 0.13$ & 10 \\
\hline V Vul........... & 1999 Aug 19 & $4500,0.0,-0.4$ & 4.6 & $7.10 \pm 0.11$ & 52 & $7.05 \pm 0.11$ & 10 \\
\hline
\end{tabular}

${ }^{\text {a }}$ The value of $T_{\text {eff }}$ is in kelvins; $\log g$ is in cgs, $[\mathrm{Fe} / \mathrm{H}]$ in dex.

b The symbol $\xi_{t}$ represents the microturbulence determined from the $\mathrm{Fe}_{\mathrm{I}}$ lines.

c The column headed $\log \epsilon$ gives the mean abundance relative to $\mathrm{H}$ (with $\log \epsilon_{\mathrm{H}}=12.00$ ). The standard deviations of the means, as calculated from the line-to-line scatter, are given. The value $n$ is the number of considered lines.

$4 \mathrm{~m}$ Blanco telescope equipped with a cross-dispersed echelle Cassegrain spectrograph and a CCD of 2048 pixels $\times 2048$ pixels. The spectrograph was set to record the wavelength interval 4900 $8250 \AA$ in 45 orders. Spectral coverage was complete between these limits. The resolving power $R \simeq 35,000$ was achieved, as measured from the Th lines in the Th-Ar comparison spectrum.

Spectra were rejected if they showed line doubling, markedly asymmetric lines, or strong emission at $\mathrm{H} \beta$. (Emission was almost always present at $\mathrm{H} \alpha$.) It is presumed that the spectra not showing these characteristics represent the atmosphere at a time when standard theoretical models may be applicable. This presumption should be tested by analysis of a series of spectra taken over the pulsational cycle. This remains to be done, but in previous papers we have analyzed several stars using spectra taken at different phases and obtained consistent results. A striking example was given in Paper III in which three observations of SS Gem gave widely different effective temperatures (4750, 5500, and $6500 \mathrm{~K}$ ) but similar results for the composition.

The abundance analyses were performed as described in earlier papers of this series. The 2002 version of the spectrum synthesis code MOOG (Sneden 1973) was used with ATLAS model atmospheres (Kurucz 1993). Molecule formation was taken into account in computing the line spectrum. Hyperfine splitting was considered for the lines of relevant atoms and lines (e.g., Sc, Mn). Atmospheric parameters were determined in the usual way from the Fe I and Fe II lines by demanding excitation and ionization equilibrium and that the iron abundance be independent of the equivalent width. Ionization equilibrium is also satisfied for $\mathrm{Si}, \mathrm{Ti}$, and $\mathrm{Cr}$ : the mean abundance difference (in dex) between neutral and ionized lines is $+0.08 \pm 0.16$ for $\mathrm{Si}$ from 11 stars, $-0.02 \pm 0.16$ for Ti from 7 stars, and $0.00 \pm 0.11$ for $\mathrm{Cr}$ from 13 stars. The adopted parameters listed in Table 2 were used for the full abundance analysis. Abundances are referred to the solar abundances given by Lodders (2003, her Table 1). Results are given in Table 3 for the stars obviously carrying the signatures of dust-gas separation and in Table 4 for the other stars.

\section{THE CHEMICAL COMPOSITIONS}

The chemical composition of an RV Tauri variable may be a blend of several signatures: (1) initial composition of the star, (2) the effects of deep mixing during stellar evolution on the composition, and (3) the effects of the dust-gas separation, and (4) if an RV Tauri star is the primary in a spectroscopic binary system, a change of composition may have resulted from mass transferred from the companion.

The initial composition may be anticipated from abundances of elements thought to be essentially unaffected in the course of evolution as a single or binary star and also by dust-gas separation. Here, $\mathrm{S}$ and $\mathrm{Zn}$ are deemed to qualify as such elements. Published abundance analyses of main-sequence stars show that a star's initial composition is generally predictable to within a small uncertainty from a determination of the abundance of one element - see Goswami \& Prantzos (2000, their Fig. 7) for a graphical summary. We adopt $\mathrm{S}$ and $\mathrm{Zn}$ as the reference elements here, from which we predict initial abundances of other elements to high accuracy.

Although the prehistory of RV Tauri variables is unknown in detail, it may be assumed that they have experienced the first dredge-up, which brings $\mathrm{CN}$-cycled material into the atmosphere. This reduces the $\mathrm{C}$ abundance and increases the $\mathrm{N}$ abundance (Iben 1967). If RV Tauri variables have evolved from the AGB on which they may have experienced thermal pulses and the third dredge-up, they are expected to be enriched in $\mathrm{C}$ and possibly also in the $s$-process elements (Busso et al. 1999). This last statement will require modification if a star has accreted substantial amounts of gas from a companion directly or through transfer from a circumbinary disk. If the RV Tauri stars are the primary of a binary, a change of composition may have resulted from mass transferred from the companion.

When gas cools and dust grains form, the abundances of the elements in the gas phase are reduced below their initial abundances. Calculations of the equilibrium distribution of an element between dust and gas have been reported by many authors 
TABLE 3

Elemental Abundances

\begin{tabular}{|c|c|c|c|c|c|c|c|c|c|c|c|c|c|c|c|c|c|c|c|}
\hline \multirow[b]{2}{*}{ SPeCIES } & \multirow[b]{2}{*}{$\log \epsilon_{\odot}^{0}$} & \multicolumn{3}{|c|}{ UY CMA } & \multicolumn{3}{|c|}{ EQ CAS } & \multicolumn{3}{|c|}{ HP LYR } & \multicolumn{3}{|c|}{ DY ORI } & \multicolumn{3}{|c|}{ LR ScO } & \multicolumn{3}{|c|}{ BZ Sct } \\
\hline & & {$[\mathrm{X} / \mathrm{H}]$} & $n$ & {$[\mathrm{X} / \mathrm{Fe}]$} & {$[\mathrm{X} / \mathrm{H}]$} & $n$ & {$[\mathrm{X} / \mathrm{Fe}]$} & {$[\mathrm{X} / \mathrm{H}]$} & $n$ & {$[\mathrm{X} / \mathrm{Fe}]$} & {$[\mathrm{X} / \mathrm{H}]$} & $n$ & {$[\mathrm{X} / \mathrm{Fe}]$} & {$[\mathrm{X} / \mathrm{H}]$} & $n$ & {$[\mathrm{X} / \mathrm{Fe}]$} & {$[\mathrm{X} / \mathrm{H}]$} & $n$ & {$[\mathrm{X} / \mathrm{Fe}]$} \\
\hline $\mathrm{C}_{\mathrm{I}} \ldots \ldots \ldots . . .$. & 8.39 & $-0.03 \pm 0.19$ & 5 & +1.26 & $-0.23 \pm 0.19$ & 3 & +0.52 & $-0.29 \pm 0.12$ & 15 & +0.69 & $+0.10 \pm 0.16$ & 16 & +2.33 & $-0.05 \pm 0.13$ & 12 & +0.22 & $+0.05 \pm 0.12$ & 20 & +0.87 \\
\hline $\mathrm{N}_{\mathrm{I}} \ldots \ldots \ldots . .$. & 7.83 & & $\ldots$ & $\ldots$ & $\ldots$ & $\ldots$ & $\ldots$ & $-0.19 \pm 0.01$ & 3 & +0.79 & $+0.54 \pm 0.01$ & 3 & +2.77 & $\ldots$ & $\ldots$ & $\ldots$ & $+0.12 \pm 0.01$ & 3 & +0.93 \\
\hline $\mathrm{O}_{\mathrm{I}} \ldots \ldots \ldots$ & 8.69 & $-0.18 \pm 0.09$ & 3 & +1.11 & $+0.04 \pm 0.04$ & 2 & +0.79 & $+0.03 \pm 0.19$ & 4 & +1.01 & $-0.20 \pm 0.14$ & 2 & +2.43 & $-0.05 \pm 0.15$ & 4 & +0.12 & $+0.06 \pm 0.15$ & 4 & +0.88 \\
\hline $\mathrm{Na}$ I........ & 6.30 & -0.44 & 1 & +0.85 & $-1.65 \pm 0.13$ & 2 & -0.90 & $-0.17 \pm 0.08$ & 4 & +0.81 & $-0.25 \pm 0.06$ & 3 & +1.98 & $-0.04 \pm 0.17$ & 4 & +0.21 & $-0.05 \pm 0.05$ & 4 & +0.77 \\
\hline $\mathrm{Mg}$ I....... & 7.55 & $-1.35 \pm 0.12$ & 4 & -0.06 & $-0.52 \pm 0.19$ & 3 & +0.23 & $-0.86 \pm 0.15$ & 4 & +0.12 & $-2.32 \pm 0.11$ & 2 & +0.09 & $-0.29 \pm 0.08$ & 2 & -0.12 & $-0.75 \pm 0.02$ & 3 & +0.07 \\
\hline $\mathrm{Al}$ I ............ & 6.46 & -1.84 & 1 & -0.55 & $\ldots$ & & & $-3.21 \pm 0.09$ & 2 & -2.23 & -3.29 & 1 & -1.06 & $-0.81 \pm 0.17$ & 3 & -0.64 & & & \\
\hline Si I ......... & 7.54 & $-0.97 \pm 0.13$ & 4 & +0.32 & $-0.26 \pm 0.10$ & 11 & +0.49 & $-0.45 \pm 0.12$ & 6 & +0.53 & -1.44 & 1 & +0.79 & $+0.07 \pm 0.10$ & 10 & +0.24 & $-0.59 \pm 0.01$ & 2 & +0.23 \\
\hline Si II ........ & 7.54 & -0.95 & 1 & +0.34 & $-0.29 \pm 0.23$ & 2 & +0.46 & $-0.58 \pm 0.20$ & 2 & +0.40 & $-1.69 \pm 0.03$ & 2 & +0.54 & -0.08 & 1 & +0.09 & $-0.73 \pm 0.15$ & 2 & +0.09 \\
\hline $\mathrm{S}_{\mathrm{I} \ldots \ldots \ldots . . .}$ & 7.19 & $-0.32 \pm 0.12$ & 5 & +0.97 & $-0.35 \pm 0.15$ & 3 & +0.40 & $+0.05 \pm 0.15$ & 8 & +1.03 & $+0.20 \pm 0.06$ & 8 & +2.43 & $+0.15 \pm 0.05$ & 4 & +0.32 & $+0.18 \pm 0.15$ & 5 & +1.00 \\
\hline $\mathrm{K}_{\mathrm{I}} \ldots \ldots \ldots$ & 5.11 & & $\ldots$ & $\ldots$ & $\ldots$ & $\ldots$ & $\ldots$ & $\ldots$ & $\ldots$ & $\ldots$ & $\ldots$ & $\ldots$ & $\ldots$ & $\ldots$ & $\ldots$ & $\ldots$ & -0.73 & 1 & +0.09 \\
\hline Са I........ & 6.34 & $-1.65 \pm 0.22$ & 4 & -0.36 & $-2.02 \pm 0.11$ & 4 & -1.27 & $-1.95 \pm 0.14$ & 4 & -0.97 & $-2.18 \pm 0.14$ & 2 & +0.05 & $-0.51 \pm 0.11$ & 6 & -0.34 & $-0.91 \pm 0.10$ & 7 & -0.09 \\
\hline Sc II ...... & 3.07 & $-2.22 \pm 0.15$ & 5 & -0.93 & -3.06 & 1 & -2.31 & -2.87 & 1 & -1.89 & -2.85 & 1 & -0.62 & $-1.30 \pm 0.08$ & 4 & -1.13 & $-1.13 \pm 0.17$ & 5 & -0.31 \\
\hline Ti I......... & 4.92 & & $\ldots$ & $\ldots$ & $-1.32 \pm 0.17$ & 2 & -0.57 & & $\ldots$ & $\ldots$ & $\ldots$ & . & 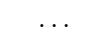 & -0.96 & 1 & -0.79 & $-1.25 \pm 0.06$ & 2 & -0.43 \\
\hline Ті пा ........ & 4.92 & $-2.38 \pm 0.13$ & 5 & -1.09 & $-1.19 \pm 0.09$ & 7 & -0.44 & $-2.97 \pm 0.19$ & 5 & -1.99 & $+2.33 \pm 0.29$ & 6 & -0.10 & $\ldots$ & $\ldots$ & $\ldots$ & $-1.18 \pm 0.12$ & 6 & -0.36 \\
\hline $\mathrm{V}_{\mathrm{I}} \ldots \ldots \ldots$ & 4.00 & 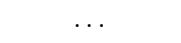 & $\ldots$ & $\ldots$ & $\ldots$ & $\ldots$ & $\ldots$ & $\ldots$ & $\ldots$ & $\ldots$ & $\ldots$ & $\ldots$ & $\ldots$ & & $\ldots$ & $\ldots$ & $\ldots$ & & \\
\hline Cr I ........ & 5.65 & $-1.88 \pm 0.11$ & 7 & -0.59 & $-2.05 \pm 0.03$ & 4 & -1.30 & $-1.24 \pm 0.16$ & 5 & -0.26 & $-1.93 \pm 0.06$ & 2 & +0.33 & $-0.28 \pm 0.05$ & 2 & -0.11 & $-0.74 \pm 0.10$ & 5 & +0.08 \\
\hline $\mathrm{Cr}$ II ........ & 5.65 & $-1.72 \pm 0.09$ & 4 & -0.43 & $-1.90 \pm 0.10$ & 3 & -1.15 & $-1.31 \pm 0.08$ & 8 & -0.33 & $-1.99 \pm 0.17$ & 8 & +0.24 & $-0.11 \pm 0.03$ & 4 & +0.06 & $-0.91 \pm 0.08$ & 9 & -0.09 \\
\hline Mn I....... & 5.50 & $-1.12 \pm 0.18$ & 5 & +0.17 & $-1.78 \pm 0.09$ & 3 & -1.03 & $-0.74 \pm 0.10$ & 4 & +0.24 & $-1.84 \pm 0.19$ & 3 & +0.37 & $-0.32 \pm 0.05$ & 2 & -0.15 & $-1.09 \pm 0.13$ & 4 & -0.27 \\
\hline $\mathrm{Fe} \ldots \ldots \ldots \ldots$ & 7.47 & -1.29 & $\ldots$ & $\ldots$ & -0.75 & $\ldots$ & $\ldots$ & -0.98 & $\ldots$ & $\ldots$ & -2.23 & $\ldots$ & $\ldots$ & -0.17 & $\ldots$ & $\ldots$ & -0.82 & $\ldots$ & $\ldots$ \\
\hline Со І......... & 4.91 & & $\ldots$ & $\ldots$ & $-0.59 \pm 0.06$ & 2 & +0.19 & $\ldots$ & $\ldots$ & $\ldots$ & $\ldots$ & $\ldots$ & $\ldots$ & & $\ldots$ & $\ldots$ & $\ldots$ & $\ldots$ & \\
\hline $\mathrm{Ni} \mathrm{I} \mathrm{.......}$ & 6.22 & $-1.57 \pm 0.10$ & 6 & -0.28 & $-0.79 \pm 0.17$ & 20 & -0.04 & $-1.14 \pm 0.12$ & 10 & -0.16 & $-2.28 \pm 0.26$ & 2 & -0.05 & $-0.46 \pm 0.17$ & 9 & -0.29 & $-0.75 \pm 0.11$ & 11 & +0.07 \\
\hline $\mathrm{Cu}$ I........ & 4.26 & $-0.75 \pm 0.20$ & 2 & +0.54 & -1.04 & 1 & -0.29 & $-0.47 \pm 0.02$ & 2 & +0.51 & $\ldots$ & $\ldots$ & 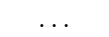 & & $\ldots$ & $\ldots$ & -0.75 & 1 & +0.07 \\
\hline $\mathrm{Zn}$ I........ & 4.63 & $-0.59 \pm 0.06$ & 3 & +0.70 & $-0.33 \pm 0.17$ & 3 & +0.46 & $-0.35 \pm 0.07$ & 4 & +0.63 & $-0.17 \pm 0.16$ & 4 & +2.06 & -0.32 & 1 & -0.15 & $+0.04 \pm 0.05$ & 3 & +0.94 \\
\hline Y II ......... & 2.20 & $-2.32 \pm 0.13$ & 4 & -1.03 & $-1.94 \pm 0.14$ & 5 & -1.19 & -2.78 & 1 & -1.80 & $-2.56 \pm 0.14$ & 3 & +0.33 & $-1.63 \pm 0.16$ & 2 & -1.46 & $-1.36 \pm 0.08$ & 4 & -0.54 \\
\hline Ва II....... & 2.18 & $-2.02 \pm 0.15$ & 3 & -0.73 & $\cdots$ & $\cdots$ &. & $-1.93 \pm 0.10$ & 2 & -0.95 & $-2.05 \pm 0.12$ & 2 & -0.18 & & $\ldots$ & $\ldots$ & -0.91 & 1 & -0.09 \\
\hline La II ....... & 1.18 & $\ldots$ & $\ldots$ & $\cdots$ & $\cdots$ & $\ldots$ & $\ldots$ & $\cdots$ & $\ldots$ & $\cdots$ & $\cdots$ & $\ldots$ & $\cdots$ & $\ldots$ & $\ldots$ & $\ldots$ & -1.79 & 1 & -0.97 \\
\hline Се пा...... & 1.61 & $\cdots$ & $\cdots$ & $\cdots$ & $\cdots$ & $\cdots$ & $\cdots$ & $\cdots$ & $\cdots$ & $\cdots$ & $\cdots$ & $\cdots$ & $\cdots$ & $\cdots$ & $\cdots$ & $\cdots$ & $-0.92 \pm 0.01$ & 2 & -0.10 \\
\hline $\operatorname{Pr}$ пा......... & 0.78 & $\ldots$ & $\ldots$ & $\cdots$ & $\cdots$ & $\cdots$ & $\cdots$ & $\cdots$ & $\cdots$ & $\cdots$ & $\cdots$ & $\cdots$ & $\cdots$ & . & $\cdots$ & $\cdots$ & $\ldots$ & 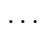 & \\
\hline $\mathrm{Nd}$ II ...... & 1.46 & $\cdots$ & $\ldots$ & $\cdots$ & $\ldots$ & $\cdots$ & $\cdots$ & $\cdots$ & $\ldots$ & $\cdots$ & $\cdots$ & $\ldots$ & $\cdots$ & $\cdots$ & $\cdots$ & .. & & $\ldots$ & \\
\hline Sm II ..... & 0.95 & & $\ldots$ & $\ldots$ & $\cdots$ & $\ldots$ & $\ldots$ & $\ldots$ & $\ldots$ & $\ldots$ & $\ldots$ & $\ldots$ & $\ldots$ & & $\ldots$ & $\ldots$ & -0.61 & 1 & +0.21 \\
\hline Eu II....... & 0.52 & -1.31 & 1 & -0.02 & $\ldots$ & $\ldots$ & $\ldots$ & $\ldots$ & $\ldots$ & $\cdots$ & $\cdots$ & $\ldots$ & $\cdots$ & -0.24 & 1 & -0.07 & -0.64 & 1 & +0.18 \\
\hline
\end{tabular}


TABLE 4

Elemental Abundances

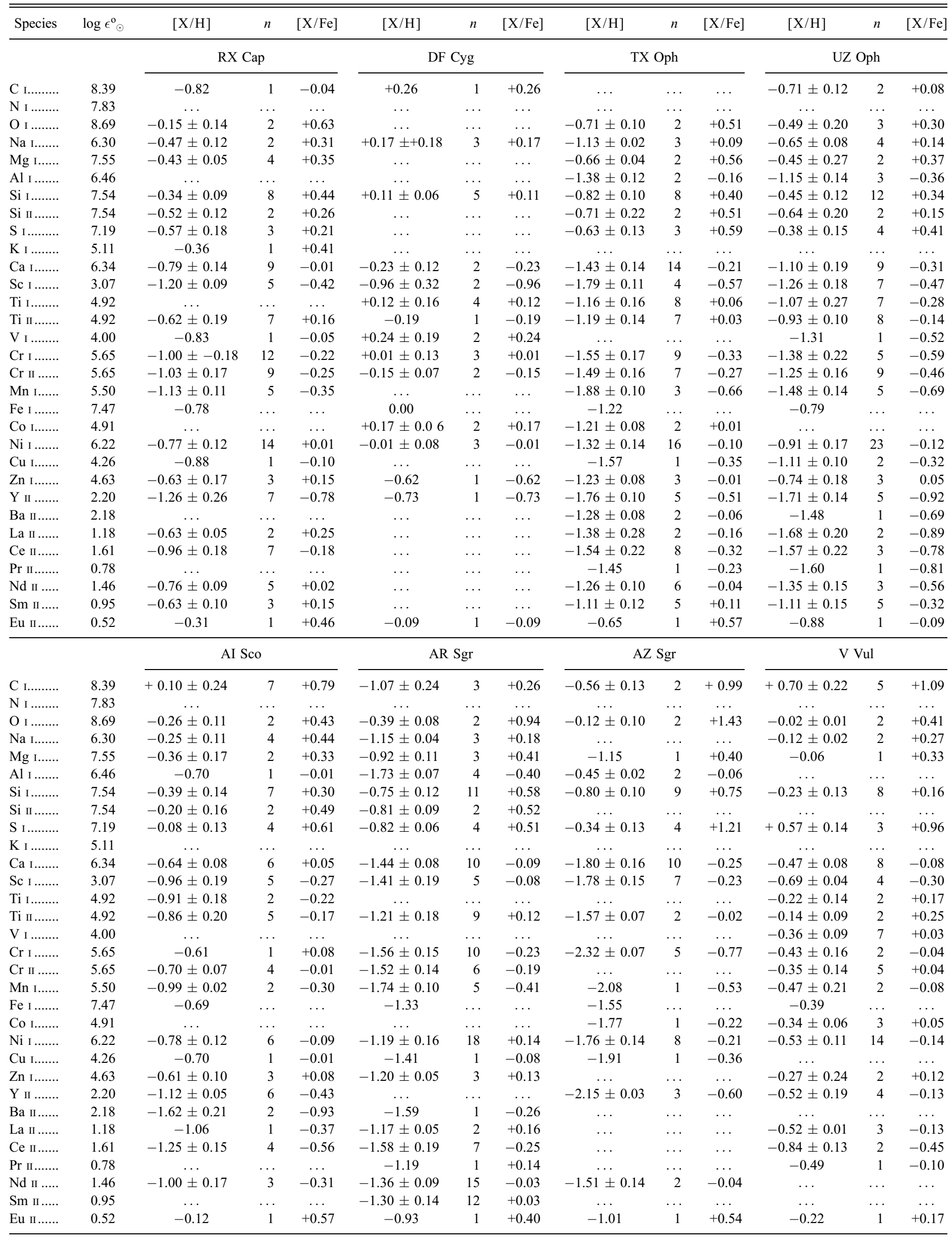


and, most recently, by Lodders (2003; her Table 1). Principal factors influencing the gas phase abundances are the temperature, pressure, and initial composition.

For elements providing major species of grains (e.g., Al and corundum- $\mathrm{Al}_{2} \mathrm{O}_{3}$ ), one may define a condensation temperature (here, $T_{C}^{\prime}$ ) at which the vapor pressure of the grain equals the partial pressure of the species in the gas. Below this condensation temperature, a species is highly underabundant in the gas. Other elements, particularly trace elements, are absorbed by major species of grains with a degree of absorption that may differ from element to element for a given grain type. This has led to the concept of a " $50 \%$ condensation temperature" - the temperature at which $50 \%$ of the trace element is in the gas and $50 \%$ in grains. We adopt the $50 \%$ condensation temperatures given by Lodders (2003) in her Table 8 for gas of solar composition (her Table 1) at a total pressure of $10^{-4}$ bar. We denote the adopted temperatures by the symbol $T_{C}$. For elements providing the major species of grains, the difference between $T_{C}^{\prime}$ and the cooler $T_{C}$ is less than $40 \mathrm{~K}$ for all but two elements- $\mathrm{Si}$ and $\mathrm{Ca}$ - which we discuss below (§ 4.1).

Our assumption is that the effects of dust-gas separation on a stellar composition will be revealed as a correlation between the underabundance of an element and that element's condensation temperature. Obvious approximations may invalidate the assumption: grain formation around the RV Tauri stars may not occur under conditions of thermodynamic equilibrium; the pressures may differ from one formation site to another; the initial composition may differ, as must certainly be the case for the high-velocity stars, from the assumed solar mix. Yet, the fact is that in many cases, the underabundances are remarkably smoothly correlated with $T_{C}$ (and $T_{C}^{\prime}$ ).

In the following subsections, we present and discuss the compositions of the stars in Table 1 but leave comment on the $\mathrm{C}, \mathrm{N}$, and $\mathrm{O}$ abundances and heavy ( $\mathrm{Y}$ to $\mathrm{Eu}$ ) elements to subsequent sections. Six stars have a composition greatly affected by dustgas separation (or another process): UY CMa, EQ Cas, HP Lyr, DY Ori, LR Sco, and BZ Sct. For the remainder of the sample, the signature of dust-gas separation is less distinct and may be absent.

\subsection{UY Canis Majoris}

Our abundance analysis ( Table 3 ) shows clearly that UY CMa is a victim of severe dust-gas separation. This is not unexpected because Preston et al. (1963) assigned it their spectroscopic class B, and our earlier analyses of class B stars found them affected by dust-gas separation. Elements of the highest $T_{C}$ (e.g., Sc and Ti) are underabundant by a factor of about 200 . The deficiencies $[\mathrm{X} / \mathrm{H}]$ are very well correlated with condensation temperature $T_{C}$ (Fig. 2). ${ }^{2}$ Judged by the abundances of $\mathrm{Na}, \mathrm{S}$, and $\mathrm{Zn}$, elements of low condensation temperature, the initial metallicity corresponds to $[\mathrm{Fe} / \mathrm{H}] \simeq-0.4$. A possibly slightly higher metallicity is suggested by the $\mathrm{O}$ abundance with the implication that even $\mathrm{Na}, \mathrm{S}$, and $\mathrm{Zn}$ may be slightly depleted. The high radial velocity suggests that UY CMa belongs to the thick disk. We have not adjusted the $[\mathrm{X} / \mathrm{H}]$ by the small amounts necessary to account for the fact that a thick-disk star of $[\mathrm{Fe} / \mathrm{H}] \simeq-0.4$ has a nonsolar mix of elements, i.e., $[\mathrm{X} / \mathrm{Y}] \neq 0$ (Reddy et al. 2003).

\section{2. $R X$ Capricorni}

Inspection of the abundances listed in Table 4 suggests that RX Cap is either a moderately metal-poor thick-disk star or a

\footnotetext{
${ }^{2}$ Usual spectroscopic notation is adopted: $[\mathrm{X} / \mathrm{Y}]=\log (\mathrm{X} / \mathrm{Y})_{\text {star }}-$ $\log (\mathrm{X} / \mathrm{Y})_{\odot}$.
}

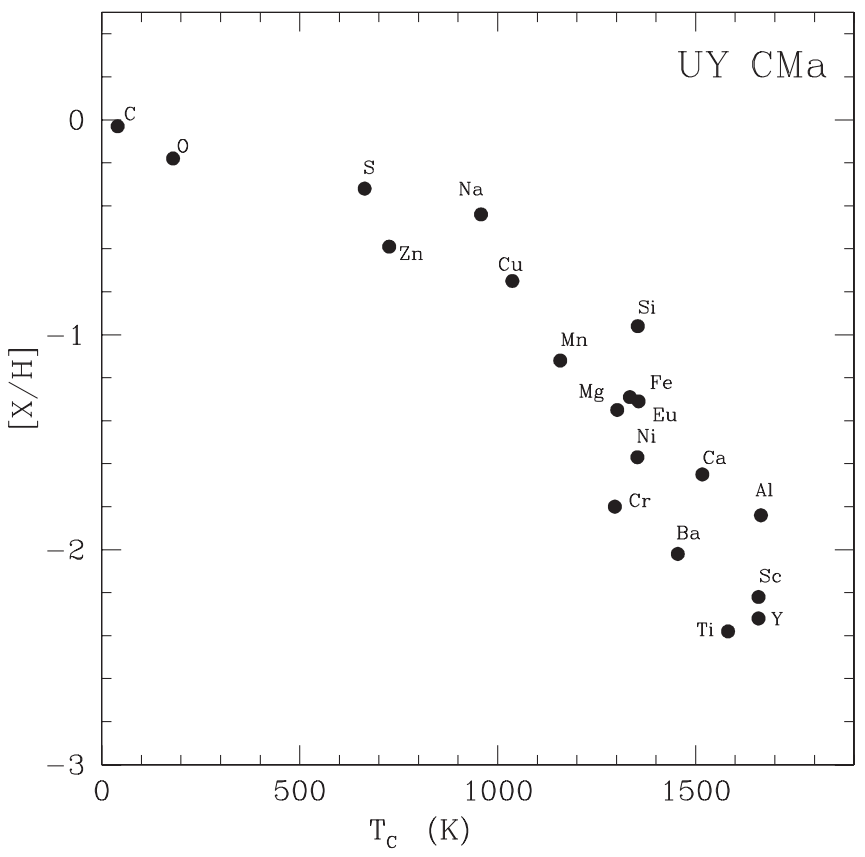

FIG. 2.-Abundance $[\mathrm{X} / \mathrm{H}]$ vs. condensation temperature $T_{C}$ for UY CMa. Elements are identified by their chemical symbol.

rather metal-rich halo star, but possessing a normal composition unaffected by dust-gas separation. The inferred initial iron abundance is $[\mathrm{Fe} / \mathrm{H}] \simeq-0.7$ from consideration of $\mathrm{S}, \mathrm{Zn}, \mathrm{Fe}$, and $\mathrm{Ni}$ abundances. Within the possible errors and especially considering that some abundances are based on a single line, a majority of the relative abundances $[\mathrm{X} / \mathrm{Fe}]$ are as expected. We note, for example, the positive $[\mathrm{X} / \mathrm{Fe}]$ for the $\alpha$-elements $\mathrm{Mg}, \mathrm{Si}, \mathrm{S}$, and $\mathrm{Ti}$, and the mild deficiency of Mn. One detects a suggestion of dust-gas separation from the low Sc abundance: $[\mathrm{Sc} / \mathrm{Fe}]=-0.4$ when $[\mathrm{Sc} / \mathrm{Fe}] \simeq 0.0$ is expected, and just possibly, from $\mathrm{Ca}$, which is slightly underabundant relative to expectation for an $\alpha$-element.

\subsection{EQ Cassiopeiae}

Three spectra of this variable were available. One was rejected because some lines showed distinct doubling and He I $5875 \AA$ was in emission. The spectrum from 1999 August 19 was crowded with lines, but unblended lines found with the help of the Arcturus spectrum are symmetric. The atmosphere at this time was quite $\operatorname{cool}\left(T_{\text {eff }}=4500 \mathrm{~K}\right)$. A useful spectrum from 2001 August 19 caught the star at a warmer phase $\left(T_{\text {eff }}=5300 \mathrm{~K}\right)$. Although $\mathrm{H} \alpha$ was in emission, unblended lines were symmetric and deemed suitable for an abundance analysis. A radial velocity of $-158 \pm$ $1 \mathrm{~km} \mathrm{~s}^{-1}$ was measured from the 1999 August and 2001 August spectra, confirming that the star is of high velocity. The two spectra provide similar results for the elemental abundances. Iron abundances from the two spectra are given in Table 2. In Table 3, we list the abundances from the 2001 spectrum. Differences in $[\mathrm{X} / \mathrm{Fe}]$ between the 2001 and 1999 spectra are in the range -0.20 to +0.14 with differences of less than \pm 0.1 for 9 of the 15 elements common to the two analyses.

There are what appear to be signatures of the dust-gas separation (Fig. 3), but the $[\mathrm{X} / \mathrm{H}]$ versus $T_{C}$ relation is not as striking and simple as that for UY CMa. Preston et al.'s (1963) assignment of the class "B?" may reflect a hint of the unusual composition differences between the star and RV B stars like UY $\mathrm{CMa}$. The abundances $[\mathrm{S} / \mathrm{H}] \simeq[\mathrm{Zn} / \mathrm{H}] \simeq-0.4$ point to an initial $[\mathrm{Fe} / \mathrm{H}]$ of -0.5 to -0.4 . The gross underabundances of $\mathrm{Ca}$ 


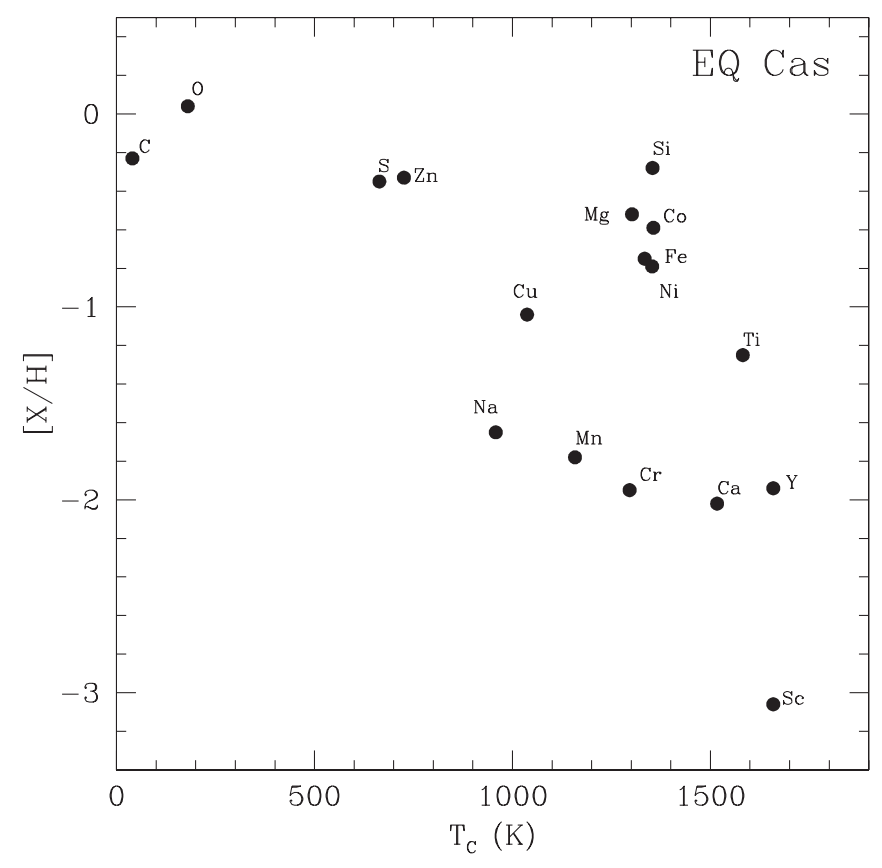

FIG. 3.- Same as Fig. 2, but for EQ Cas.

and $\mathrm{Sc}([\mathrm{Ca} / \mathrm{H}]=-2.0$ and $[\mathrm{Sc} / \mathrm{H}]=-3.1)$ point to dust-gas separation. Yet, among the RV Tauri variables analyzed by us in this series of papers, EQ Cas is unique in showing additional anomalies. In particular, the $\mathrm{Na}$ abundance is remarkably low: $[\mathrm{Na} / \mathrm{Fe}]=-0.9$, where all other RV Tauri variables, even those severely affected by dust-gas separation, show a positive $[\mathrm{Na} / \mathrm{Fe}]$. Furthermore, our estimate for $[\mathrm{Na} / \mathrm{Fe}]$ may be an upper limit because the $\mathrm{Na}$ I lines are very weak. The other anomalies are the low values of $[\mathrm{Cr} / \mathrm{H}],[\mathrm{Mn} / \mathrm{H}]$, and $[\mathrm{Cu} / \mathrm{H}]$ and the high value of $[\mathrm{Ti} / \mathrm{H}]$ relative to other elements of similar $T_{C}$. These outstanding anomalies are shown by the two independent analyses of the star except that the $\mathrm{Cu}$ I lines were not observed in the 1999 spectrum. Kameswara Rao \& Reddy (2005) show that EQ Cas abundances are tightly correlated with an element's first ionization potential (FIP). This suggests that a mechanism other than dust-gas separation has affected the photospheric abundances.

\subsection{DF Cygni}

Two spectra were acquired, but only one had symmetric unblended lines suitable for an abundance analysis (Table 4). Line selection was made by reference to the spectrum of Arcturus, but given the crowded spectrum, some key elements (e.g., Al and S) proved undetectable, and many others are represented by no more than one or two lines. The iron abundance is close to solar. Setting aside elements represented by just one line, the sole apparent anomaly is $\mathrm{Sc}([\mathrm{Sc} / \mathrm{H}]=[\mathrm{Sc} / \mathrm{Fe}]=-1.0$, not 0.0$)$. The $\mathrm{Sc}$ underabundance is suggestive of the onset of dust-gas separation but, as for other stars in Table 4, Sc (and Ca in some stars) is the only indicator of this effect. This isolation of Sc leads one to wonder about other possible explanations, e.g., non-LTE (NLTE) effects.

\subsection{HP Lyr}

This star came to our attention through Graczyk et al.'s (2002) report of it as "possibly the hottest RV Tau-type object." These authors estimated the effective temperature to be about $7700 \mathrm{~K}$. Our spectrum corresponds to $T_{\text {eff }}=6300 \mathrm{~K}$. The radial velocity of $-107 \mathrm{~km} \mathrm{~s}^{-1}$ indicates that HP Lyr is a high-velocity star. The intrinsic metallicity of the star as assessed from $\mathrm{S}, \mathrm{Zn}$, and $\mathrm{Na}$ is

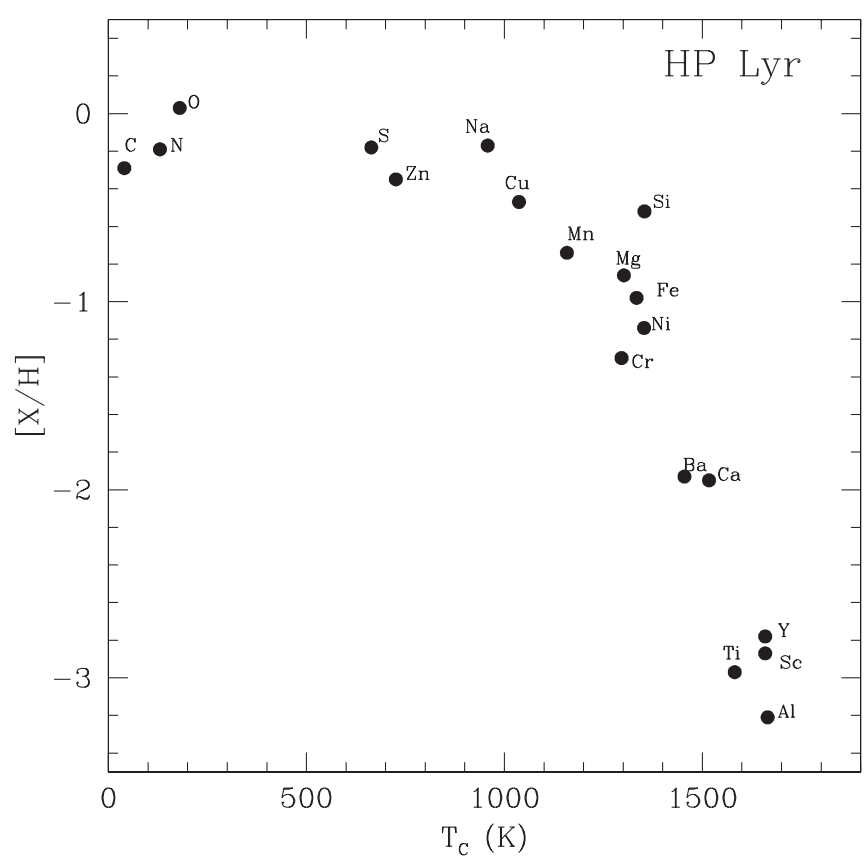

FIG. 4.-Same as Fig. 2, but for HP Lyr.

slightly subsolar $([\mathrm{Fe} / \mathrm{H}] \simeq-0.2)$. We suppose that HP Lyr may belong to the thick disk. The abundances (Table 3 and Fig. 4) show the signature of dust-gas separation. The correlation between $[\mathrm{X} / \mathrm{H}]$ and $T_{C}$ is tight, with almost no scatter in excess of that attributable to the observational errors in $[\mathrm{X} / \mathrm{H}]$.

\subsection{TX Ophiuchi}

Most elements (Table 4) have the abundance expected of a normal star with $[\mathrm{Fe} / \mathrm{H}]=-1.2$, the measured iron abundance. This metallicity and the high radial velocity suggest that the star belongs to the Galactic thick disk. One notes the normal $[\mathrm{Na} / \mathrm{Fe}]$ and $[\mathrm{Zn} / \mathrm{Fe}]$ but observes that $\mathrm{Ca}, \mathrm{Sc}$, and Ti, elements of high condensation temperature, are apparently underabundant with $[\mathrm{Ca} / \mathrm{Fe}],[\mathrm{Sc} / \mathrm{Fe}]$, and $[\mathrm{Ti} / \mathrm{Fe}]$ by about $0.5,0.6$, and $0.3 \mathrm{dex}$, respectively, below the expected initial value for a thick-disk star of $[\mathrm{Fe} / \mathrm{H}]=-1.2$. Aluminum, also of high condensation temperature, however, has its expected initial abundance.

A comparison with RX Cap reveals that the abundances scale with the difference in $[\mathrm{Fe} / \mathrm{H}]$ of 0.4 dex between the two stars. The mean difference in $[\mathrm{X} / \mathrm{H}]$ in the sense of TX Oph minus RX Cap is 0.5 dex from 20 elements with an element-to-element scatter consistent with the measurement errors. This consistency extends to $\mathrm{Ca}$, Ti, and $\mathrm{Sc}$.

\subsection{UZ Ophiuchi}

Two spectra are suitable for an abundance analysis. The spectrum from the $2.7 \mathrm{~m}$ telescope taken on 2002 June 30 is our primary source. An earlier spectrum from 2001 July 13 taken on the $2.1 \mathrm{~m}$ with the Sandiford Cassegrain echelle (McCarthy et al. 1993) provided coverage of the interval 4500-5200 $\AA$. Since most of the elements were covered in 2002 spectrum and abundances derived at two epochs are in good agreement, we chose to use only 2002 estimates. The radial velocities of $-95.1 \pm$ $0.9 \mathrm{~km} \mathrm{~s}^{-1}$ in 2001 July and $-90.2 \pm 0.8 \mathrm{~km} \mathrm{~s}^{-1}$ in 2002 June suggest membership of the Galactic thick disk for UZ Oph. Abundances are given in Table 4.

The composition resembles that of RX Cap, a star of similar metallicity and one that we described as essentially possessing its initial composition. Relative to RX Cap, the mean abundance 


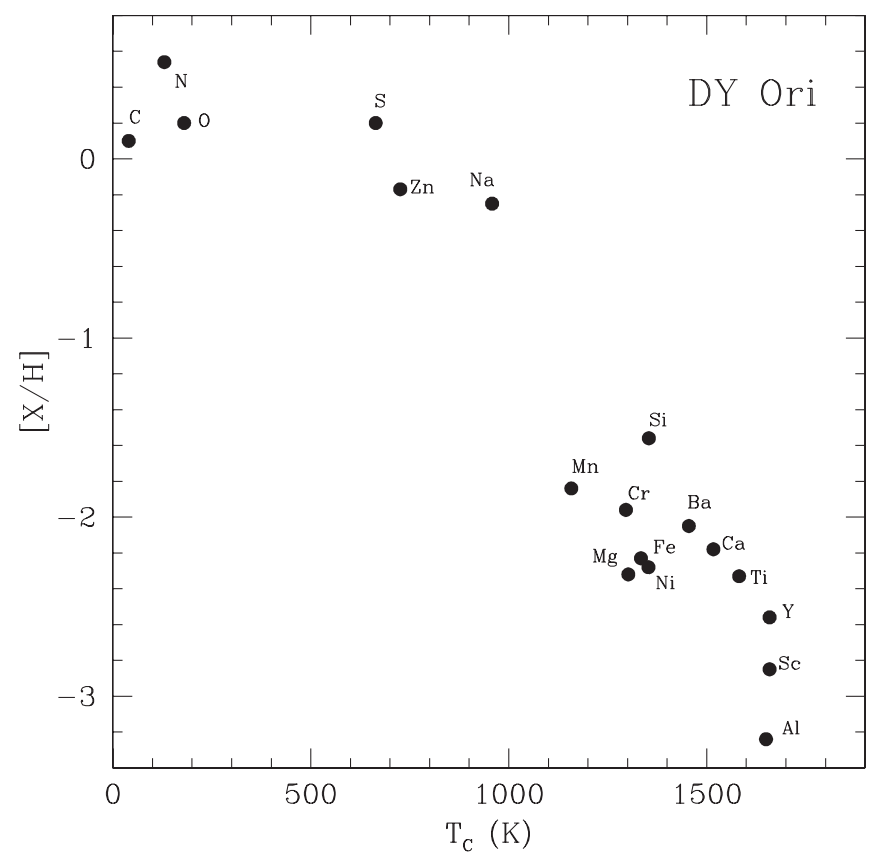

FIG. 5.-Same as Fig. 2, but for DY Ori.

difference between UZ Oph and RX Cap is $\Delta[\mathrm{X} / \mathrm{H}]=-0.15$ from 13 elements from $\mathrm{Na}$ to $\mathrm{Zn}$ with no element having a $\Delta$ outside the errors of measurement. The six heavy elements $\mathrm{Y}, \mathrm{La}$, $\mathrm{Ba}, \mathrm{Ce}, \mathrm{Nd}, \mathrm{Sm}$, and $\mathrm{Eu}$ do provide a significantly different result: $\Delta=-0.63$ : UZ Oph is relatively underabundant in $\mathrm{Y}$ to Sm but not in Eu relative to RX Cap and the expected initial composition.

\subsection{DY Orionis}

DY Orionis was analyzed in Paper II. Our reanalysis uses a new spectrum providing greater wavelength coverage, which enabled us to cover more elements relative to the earlier study. Our new results are in good agreement with the earlier results. The star, as noted in Paper II, is severely affected by dust-gas separation (Table 3 and Fig. 5). Particularly striking is the sharp onset of depletion for elements with $T_{C}$ above $1100 \mathrm{~K}$ : Na is barely depleted with $[\mathrm{Na} / \mathrm{Fe}]$ of -0.3 but $\mathrm{Si}, \mathrm{Mn}$, and $\mathrm{Cr}$ have $[\mathrm{X} / \mathrm{H}] \simeq-1.9$.

\subsection{AI Scorpii}

The abundance analysis (Table 4) implies that AI Sco's composition is that expected of a star with the measured $[\mathrm{Fe} / \mathrm{H}]=$ -0.7 . The apparent anomalies are a mild overabundance of $\mathrm{Na}$ and $\mathrm{S}$ and an underabundance of $\mathrm{Ca}, \mathrm{Sc}$, and Ti. Relative to RX Cap, $\mathrm{Na}$ to $\mathrm{Zn}$ are slightly overabundant: $\Delta=+0.13$ from 13 elements. The heavy elements $-\mathrm{Y}$ to $\mathrm{Nd}-$ are underabundant: $\Delta=-0.13$ from five elements. Europium has a normal abundance. These small differences between AI Sco and RX Cap could be attributed to the inevitable observational errors.

\subsection{LR Scorpii}

This star was analyzed previously (Giridhar et al. 1992) and discussed as a victim of dust-gas separation in Paper V. Our new analysis is summarized in Table 3. Maas (2003) has presented an abundance analysis based on spectra obtained in 2000 and 2001 when the spectroscopically estimated parameters $\left(T_{\text {eff }}, \log g\right)$ were $(6250,0.5)$ and $(5250,0.0)$, respectively. His abundances agree well with ours, especially for $[\mathrm{X} / \mathrm{Fe}]$.

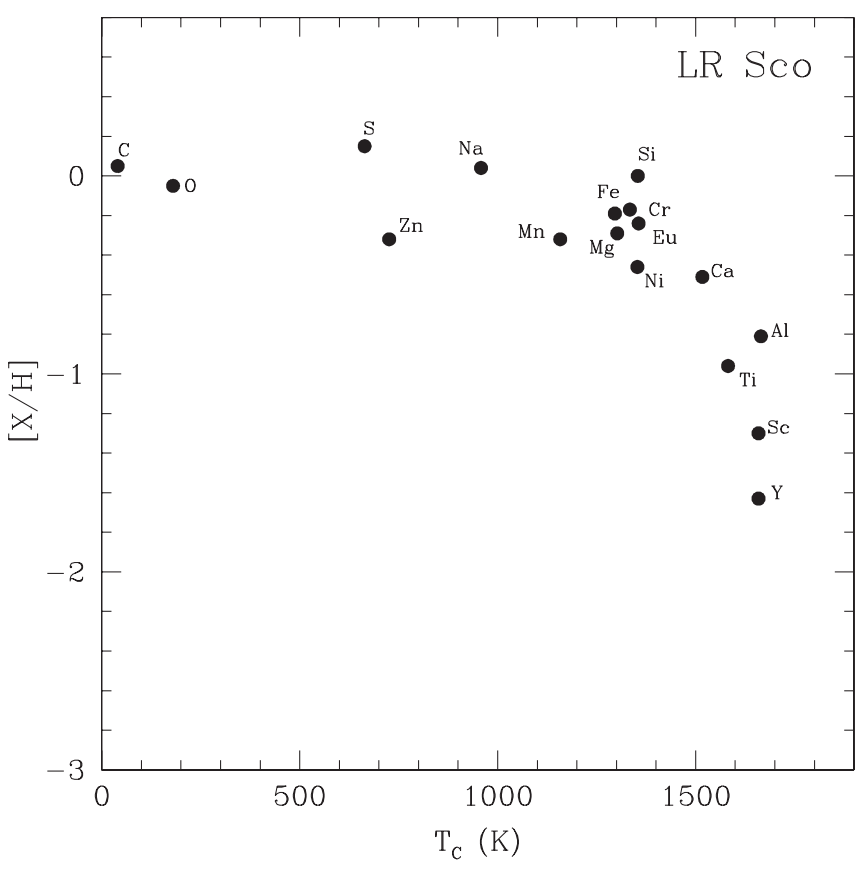

FIg. 6.-Same as Fig. 2, but for LR Sco.

Elements with $T_{C} \lesssim 1400 \mathrm{~K}$ suggest an initial iron abundance $[\mathrm{Fe} / \mathrm{H}]=-0.2$, which, with the low radial velocity, indicates that LR Sco is a member of the Galactic thin disk. Our abundance analysis (Table 3 and Fig. 6) shows that elements with a condensation temperature higher than $1600 \mathrm{~K}$ are clearly underabundant, for which $[\mathrm{X} / \mathrm{H}] \simeq-0.2$ for all elements is expected.

Our abundances $[\mathrm{X} / \mathrm{H}]$ are generally in good agreement with the 1992 results obtained from a 1989 spectrum but for $\mathrm{Al}, \mathrm{Sc}, \mathrm{Ti}$, and $\mathrm{Y}$ our results differ from the 1992 values by $-0.4,-0.4$, -0.7 , and -1.5 dex, respectively. We note that this quartet is made up of elements with $T_{C}$ of about $1600 \mathrm{~K}$. Would it be correct to ascribe the differences between our present and previous results to a growth of the depletion of the highest $T_{C}$ elements abundance? According to Lodders \& Fegley (1998), the initial condensates of the five elements are $\mathrm{Al}_{2} \mathrm{O}_{3}, \mathrm{CaAl}_{12} \mathrm{O}_{19}$, $\mathrm{Sc}_{2} \mathrm{O}_{3}, \mathrm{CaTiO}_{3}$, and $\mathrm{Y}_{2} \mathrm{O}_{3}$. This mix precludes a substantial depletion of $\mathrm{Ca}$. Calcium with $T_{C}=1505 \mathrm{~K}$ gave the same result $([\mathrm{Ca} / \mathrm{H}]=-0.5)$ from the 1989 and 2002 spectra. Maas (2003) abundances may be consistent with the suspected lowering of the abundances of $\mathrm{Al}, \mathrm{Sc}, \mathrm{Ti}$, and $\mathrm{Y}$ between 1989 and 2002, but a uniform analysis of all available spectra is desirable. Observations of LR Sco should be continued.

\subsection{AR Sagittarii}

The abundance analysis is summarized in Table 4. This highvelocity star shows no convincing evidence for a dust-gas separation. The initial metallicity is identified as $[\mathrm{Fe} / \mathrm{H}] \simeq-1.2$. Relative to TX Oph, a star of the same measured $[\mathrm{Fe} / \mathrm{H}]$, the mean difference is 0.0 from 14 elements from $\mathrm{Na}$ to $\mathrm{Zn}$, and -0.12 from 7 elements from Ba to Eu. In short, AR Sgr and TX Oph are of essentially identical normal composition.

\subsection{AZ Sagittarii}

The star was in a cool phase at the time of observation and the spectrum is crowded with lines. The abundance analysis ( Table 4) is based on few lines. The available spectrum did not go shortward of about $4900 \AA$ and, therefore, the blue and useful $\mathrm{Zn}$ I lines were not recorded. Our spectrum gives the radial velocity as $-110 \mathrm{~km} \mathrm{~s}^{-1}$. This velocity identifies AZ Sgr as a high-velocity star. 


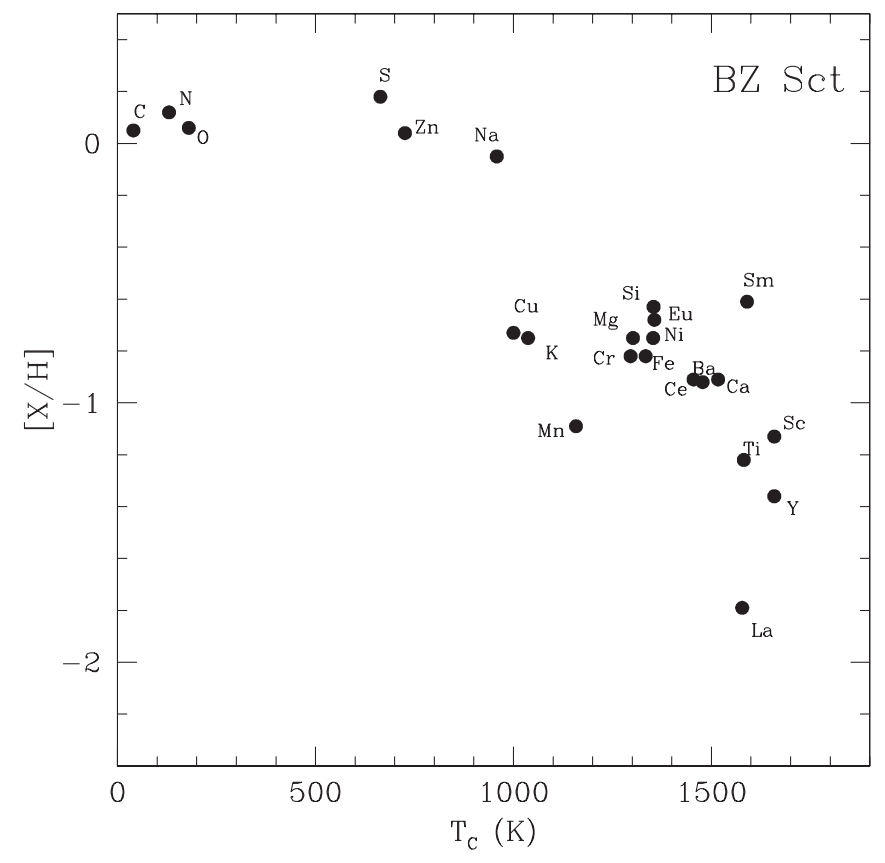

FIG. 7.- Same as Fig. 2, but for BZ Sct.

Judged with respect to a normal star of the measured $[\mathrm{Fe} / \mathrm{H}]=$ $-1.6, \mathrm{AZ}$ Sgr's anomalies are a high $\mathrm{S}$ abundance and a low $\mathrm{Cr}$ abundance: $[\mathrm{S} / \mathrm{H}]$ is too high by about $0.8 \mathrm{dex}$ and $[\mathrm{Cr} / \mathrm{H}]$ is too low by about $0.8 \mathrm{dex}$. The $\mathrm{S}$ abundance is based on weak lines and is sensitive to the adopted effective temperature. The $\mathrm{Cr}$ abundance appears to be securely based, as it is derived using five neutral Cr lines of moderate strengths. Relative to TX Oph and AR Sgr, stars with a slightly higher measured $[\mathrm{Fe} / \mathrm{H}]$, the $\mathrm{S}$ overabundance is less marked, but the $\mathrm{Cr}$ underabundance remains distinctive. Pending acquisition and analysis of additional spectra of AZ Sqr, preferably at a warmer phase, we adopt the conclusion that this star is a metal-poor high-velocity star unaffected by dustgas separation. The $\mathrm{Cr}$ underabundance is unexplained.

\subsection{BZ Scuti}

This star's composition (Table 3 and Fig. 7) shows evidence of dust-gas separation. $\mathrm{Na}, \mathrm{S}$, and $\mathrm{Zn}$ abundances show that BZ Sct's initial metallicity was close to solar. These abundances are based on unblended lines in the spectrum of this warm RV Tauri star. Elements with a condensation temperature higher than about $1200 \mathrm{~K}$ are depleted by a factor of about 8 with an element-to-element scatter comparable to the errors of measurement. There is a hint that elements with $T_{C}$ greater than $1600 \mathrm{~K}$ are more severely depleted. The extreme depletion of $\mathrm{La}$ is based on a single line.

\subsection{Vulpeculae}

This star is clearly $\mathrm{C}$-rich. The carbon enrichment derived from $\mathrm{C}_{\mathrm{I}}$ lines is also signaled by the presence of $\mathrm{C}_{2} \mathrm{Swan}$ bands. Apart from carbon and possibly sulfur, the composition of V Vul resembles closely that of the other mildly Fe-poor stars in Table 4. The mean difference, for example, relative to RX Cap is +0.40 for 11 elements from $\mathrm{Na}$ to $\mathrm{Zn}$, and +0.27 for 4 elements from $\mathrm{Y}$ to $\mathrm{Eu}$, where the $[\mathrm{Fe} / \mathrm{H}]$ difference is +0.4 also.

\section{THE COMPOSITIONS OF RV TAURI STARS}

\subsection{Abundance Anomalies and Condensation Temperatures}

In recent papers on RV Tauri compositions, abundance versus condensation temperature plots have been constructed with values of $T_{C}$ taken from Lodders \& Fegley (1998), which are also $50 \%$ condensation temperatures. Here, we adopt $T_{C}$ from Lodders (2003) (see $\S 3$ ). The effect of a substitution of the 2003 for the 1998 estimates of $T_{C}$ is in most cases a reduction in the scatter of $[\mathrm{X} / \mathrm{H}]$ about the mean trend, with $T_{C}$ for those stars obviously affected by the dust-gas separation. In our present sample, this is certainly the case for UY CMa, HP Lyr, DY Ori, and LR Sco. The elements for which replacement of the 1998 by the $2003 T_{C}$ estimates reduces their status as outliers in the $[\mathrm{X} / \mathrm{H}]$ versus $T_{C}$ plot are notably $\mathrm{Si}$ and $\mathrm{Ba}$. In the case of $\mathrm{Si}, T_{C}$ is lowered to 1302 from $1529 \mathrm{~K}$ in 1998 . The $T_{C}$ for $\mathrm{Ba}$ is raised from $1162 \mathrm{~K}$ in 1998 to $1447 \mathrm{~K}$ in 2003 . For other elements we measured the change in $T_{C}$ from the 1998 and 2003 estimates differs by less than about $50 \mathrm{~K}$, except for $\mathrm{Ca}$ and $\mathrm{Cu}$ for which the 2003 values are lower by $130-140 \mathrm{~K}$, too small of a change to affect the appearance of a $[\mathrm{X} / \mathrm{H}]$ versus $T_{C}$ correlation.

It is useful to look behind the $T_{C}$ estimates at the highest temperature condensates expected in equilibrium for solar composition gas. For this exercise, Lodders (2003) provides the details. Our discussion considers the development of condensates as gas is cooled in equilibrium. As long as equilibrium is maintained, the discussion is readily reworded to consider the dissolution of condensates as the gas-dust mixture is heated.

Inspection of Figures 2, 4, 6, and 7 show that the four most depleted elements are $\mathrm{Al}, \mathrm{Sc}, \mathrm{Ti}$, and $\mathrm{Y}$, with very similar $T_{C}$ $(\simeq 1640 \mathrm{~K})$. The initial condensates are $\mathrm{Al}_{2} \mathrm{O}_{3}$ and $\mathrm{CaTiO}_{3}$ leading at a slightly lower temperature to the major condensates $\mathrm{CaAl}_{12} \mathrm{O}_{19}$ (hibonite) and $\mathrm{CaTi}_{3} \mathrm{O}_{10}, \mathrm{Ca}_{3} \mathrm{Ti}_{2} \mathrm{O}_{7}$, and $\mathrm{Ca}_{4} \mathrm{Ti}_{3} \mathrm{O}_{10}$ (three forms of calcium titanate). Calcium titanate may severely deplete gas of Ti but not $\mathrm{Ca}$; the initial $\mathrm{Ca} / \mathrm{Ti}$ ratio is about 20 . Similarly, if hibonite scours $\mathrm{Al}$ from the gas, little $\mathrm{Ca}$ is removed and the initial ratio $\mathrm{Ca} / \mathrm{Al} \simeq 1$ is increased to $\mathrm{Ca} / \mathrm{Al} \simeq 10$. Sc and $\mathrm{Y}$ are depleted as $\mathrm{Sc}_{2} \mathrm{O}_{3}$ and $\mathrm{Y}_{2} \mathrm{O}_{3}$ dissolve in hibonite.

Calcium and $\mathrm{Ba}$ are lost to grains at a slightly lower $T_{C}$. The minor loss of $\mathrm{Ca}$ from the gas to hibonite is enhanced by the condensation of $\mathrm{Ca}_{2} \mathrm{Al}_{2} \mathrm{SiO}_{7}$ (gehlenite) leading to $\mathrm{Ca} / \mathrm{Al} \sim 1$ in the gas with both $\mathrm{Ca}$ and $\mathrm{Al}$ depleted for $T_{C} \simeq 1500 \mathrm{~K}$. Barium is removed from the gas when $\mathrm{BaTiO}_{3}$ dissolves in calcium titanate. In the 1998 calculations, Ba was identified as dissolving in $\mathrm{CaTiO}_{3}$ (perovskite), but in the 2003 calculations it was considered to dissolve into forms of calcium titanate. It is interesting that although the mode of transfer of $\mathrm{Ca}$ and $\mathrm{Ba}$ to condensates differs, their depletion $[\mathrm{X} / \mathrm{H}]$ in the gas is generally quite similar for stars severely affected by dust-gas separation. (Rare earths- $\mathrm{La}$ to $\mathrm{Eu}$ in our analyses - dissolve in hibonite and titanate.)

Descending the $T_{C}$ scale, we encounter $\mathrm{Mg}$ and Si. Magnesium is removed from the gas primarily through $\mathrm{Mg}_{2} \mathrm{SiO}_{4}$ (forsterite) with $\mathrm{MgAl}_{2} \mathrm{O}_{4}$ (spinel) also contributing. If this were the sole way to remove $\mathrm{Mg}$ and $\mathrm{Si}$ from the gas, the $\mathrm{Si} / \mathrm{Mg}$ ratio in the gas would swing from $\mathrm{Si} / \mathrm{Mg} \simeq 1$ to $\simeq 2$. The $T_{C}$ for Si listed by Lodders \& Fegley (1998) is the temperature at which gehlenite $\left(\mathrm{Ca}_{2} \mathrm{Al}_{2} \mathrm{SiO}_{7}\right)$ forms, but because $\mathrm{Ca}$ and $\mathrm{Al}$ are at least an order of magnitude less abundant than $\mathrm{Si}$, this condensate removes very little silicon from the gas. At a slightly lower temperature, $\mathrm{MgSiO}_{3}$ (enstatite) condenses and then one expects $\mathrm{Si} / \mathrm{Mg} \sim 1$ and similar depletions of $\mathrm{Mg}$ and $\mathrm{Si}$. The $T_{C}$ taken from Lodders (2003) refers to condensation of enstatite.

\subsection{Depletion Patterns}

The stars in this and earlier papers of our series exhibiting dust-gas separation seem to form a family with a single outcast. The main characteristic of the family is an ordering of depletions $[\mathrm{X} / \mathrm{H}]$ by the condensation temperature $T_{C}$. In our present 


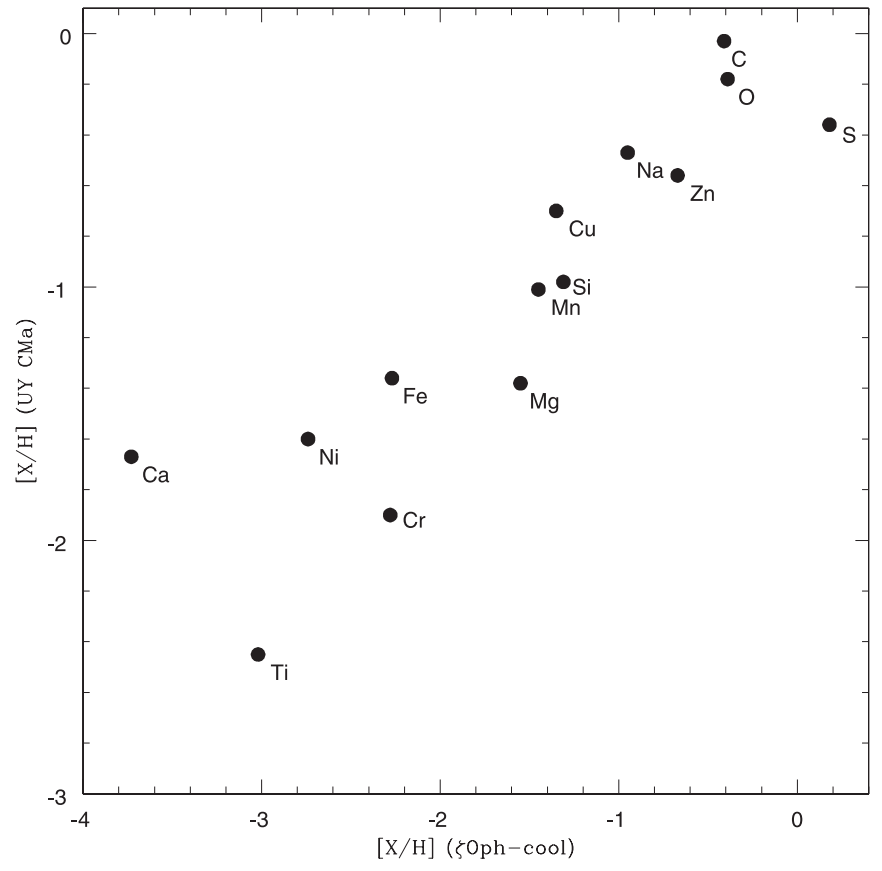

FIG. 8.-Abundance $[\mathrm{X} / \mathrm{H}]$ for UY CMa vs. $[\mathrm{X} / \mathrm{H}]$ for the cool interstellar cloud along the line of sight to $\zeta$ Oph. Elements are identified by their chemical symbol.

sample, the extremes are marked by LR Sco and the pair HP Lyr and DY Ori. LR Sco would be deemed to have a normal composition but for depletions $([\mathrm{X} / \mathrm{H}] \sim-1)$ of $\mathrm{Al}, \mathrm{Sc}, \mathrm{Ti}$, and $\mathrm{Y}$, the elements of the highest $T_{C}$. At the other extreme, all elements with $T_{C} \geq 1200 \mathrm{~K}$ show a depletion increasing with $T_{C}$ and attaining a level $[\mathrm{X} / \mathrm{H}] \sim-3$ at the highest $T_{C}$. This is not a single parameter family; see, for example, the differences in $[\mathrm{X} / \mathrm{H}]$ between the extremes HP Lyr and DY Ori.

BZ Sct appears not to show the family's smooth run of $[\mathrm{X} / \mathrm{H}]$ with $T_{C}$, but the several outliers $(\mathrm{Cu}, \mathrm{K}, \mathrm{Sm}$, and $\mathrm{La})$ in Figure 7 are based on a single line. If this is deemed a possible reason for the scatter, one is left with just $\mathrm{Mn}$ as showing a slightly discrepant $[\mathrm{X} / \mathrm{H}]$. R Scuti was considered quite anomalous by Luck (1981), but in Paper V we showed that the underabundant elements, including the heavy elements analyzed by Luck, were among those with the highest $T_{C}$. With due allowance for errors in the abundance determinations, differences in initial abundances as a function of metallicity and population class (i.e., thick versus thin disk), and small familial differences, all analyzed stars but one may be said to belong to the same family bounded by LR Sco and HP Lyr-DY Ori. ${ }^{3}$

The sole nonmember of the family is EQ Cas, for which the upper envelope to the run of $[\mathrm{X} / \mathrm{H}]$ with $T_{C}$ resembles the pattern shown by HP Lyr, but several elements fall well below this envelope: notably, Na, Mn, and Cr. Kameswara Rao \& Reddy (2005) show that the $[\mathrm{X} / \mathrm{H}]$ values for EQ Cas are well correlated with an element's FIP (see below).

\subsection{Interstellar Depletions}

The abundances for stars affected by dust-gas separation correlate quite well with those for the cool interstellar gas toward $\zeta$ Oph (Savage \& Sembach 1996) (Fig. 8). Depletions of elements in interstellar gas are attributed to incorporation of the

\footnotetext{
3 Here, we note a good correspondence between R Sct and LR Sco. It would be useful to extend the analysis of LR Sco to more heavy elements: Paper V gave $[\mathrm{X} / \mathrm{H}]$ for nine elements between $\mathrm{Y}$ and Eu but Table 3 includes just two.
}

depleted element into and onto grains. The grains may have formed primarily in denser regions of interstellar clouds, but, as the detection and analysis of grains in meteorites demonstrates, some grains were formed in outflows of red giants, e.g., stellar ejecta from supernovae and novae shells. Grain formation and depletion of atoms in the gas in the interstellar medium cannot be expected to mimic very closely the convolution of grain formation, depletion of gas in the dusty reservoir, separation of gas from dust, accretion of the gas, and mixing of the gas with the stellar atmosphere. Despite these caveats, the close correspondence between the affected RV Tauri stars and the interstellar gas is suggestive of a similarity between the physical processes involved. The differences between the stellar and interstellar depletions may be clues to the physical processes at work.

\subsection{The Initial Fe Abundances}

On the assumption that $\mathrm{S}$ and $\mathrm{Zn}$ are unaffected by dust-gas depletion, their abundances may be taken to be a star's initial abundances and hence used to provide estimates of the initial abundance of $\mathrm{Fe}$ and other elements. S and $\mathrm{Zn}$ are the sampled elements with the lowest $T_{C}$ that are considered to be unaffected by internal nucleosynthesis and mixing. The run of $\mathrm{S}$ and $\mathrm{Zn}$ abundances with $\mathrm{Fe} / \mathrm{H}$ is known for unevolved stars (Mishenina et al. 2002; Nissen et al. 2004; Prochaska et al. 2000; Ryde \& Lambert 2004). Here we are interested in the abundances of thin and thick-disk stars for which differences in $[\mathrm{S} / \mathrm{Fe}]$ and $[\mathrm{Zn} / \mathrm{Fe}]$ at a given $[\mathrm{Fe} / \mathrm{H}]$ are small, say, about 0.1 dex with the larger values found for the thick-disk stars. In successive figures we show $[\mathrm{Zn} / \mathrm{Fe}]$ and $[\mathrm{S} / \mathrm{Fe}]$ versus $[\mathrm{Fe} / \mathrm{H}]$ (Figs. 9 and 10 ), and $[\mathrm{S} / \mathrm{Zn}]$ versus $[\mathrm{Zn} / \mathrm{H}]$ (Fig. 11). Stars with a radial velocity of greater than $100 \mathrm{~km} \mathrm{~s}^{-1}$ are termed "high velocity" and shown by filled symbols in the figures.

In Figure 9, we show $[\mathrm{Zn} / \mathrm{Fe}]$ versus $[\mathrm{Fe} / \mathrm{H}]$ for all of the $\mathrm{RV}$ Tauri variables considered in this and previous papers in this series. Results for additional RV Tauri variables are taken from

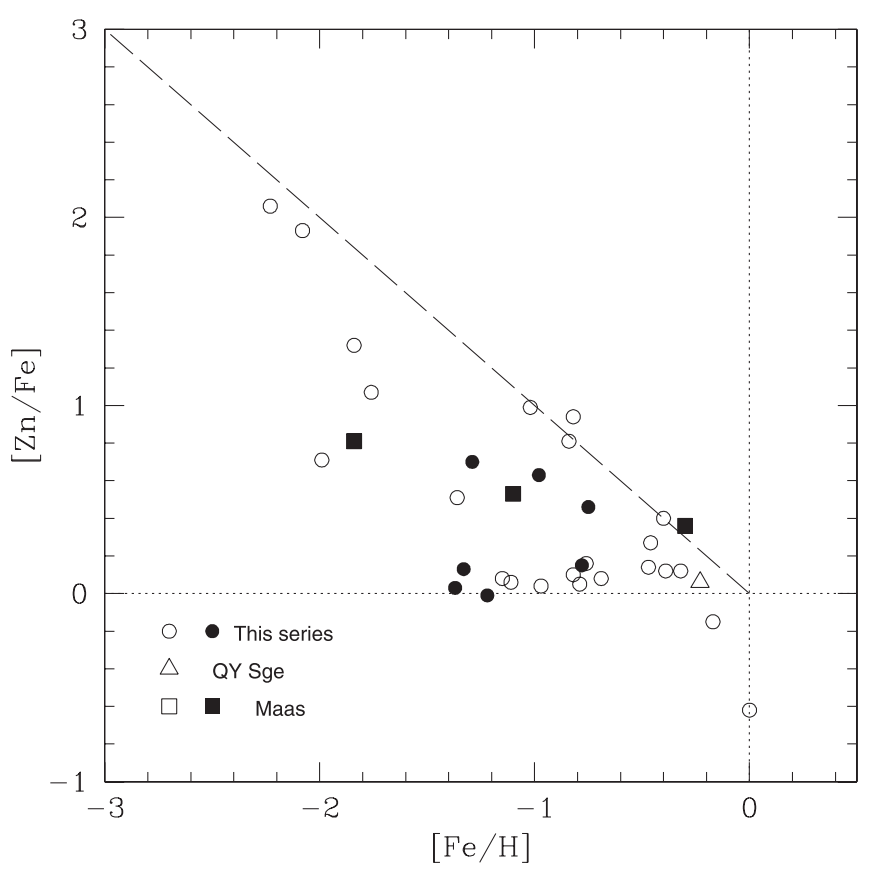

FIG. 9.- Abundance ratio $[\mathrm{Zn} / \mathrm{Fe}]$ vs. abundance $[\mathrm{Fe} / \mathrm{H}]$ for RV Tauri and related variables. RV Tauri stars from our series of papers are represented by circles. QY Sge (Kameswara Rao et al. 2002) is shown by the triangle. RV Tauri stars analyzed by Maas et al. (2002) and Maas (2003) are shown by squares. Filled circles and squares denote high-velocity stars. 


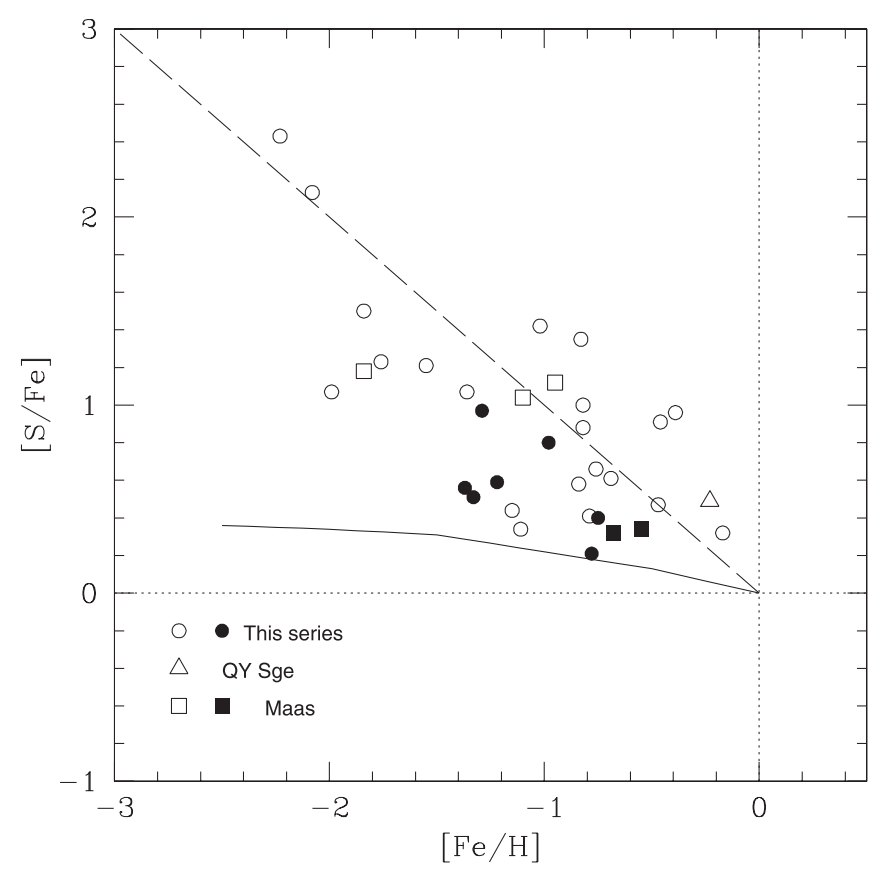

FIG. 10.-Abundance ratio $[\mathrm{S} / \mathrm{Fe}]$ vs. abundance $[\mathrm{Fe} / \mathrm{H}]$ for $\mathrm{RV}$ Tauri and related variables. RV Tauri stars from our series of papers are represented by circles. QY Sge (Kameswara Rao et al. 2002) is shown by the triangle. RV Tauri stars analyzed by Maas et al. (2002) and by Maas (2003) are shown by squares. Filled circles and squares denote high-velocity stars. The solid line shows the run of $[\mathrm{S} / \mathrm{Fe}]$ with $[\mathrm{Fe} / \mathrm{H}]$ for unevolved stars (see text).

Maas et al. (2002) for RU Cen and SX Cen, and Maas (2003) for IRAS 09538-7622, 16230-3410, 17038-4815, and 172334330. We have added a point for QY Sge (Kameswara Rao et al. 2002), which, on circumstantial evidence, was described as a dustobscured RV Tauri variable. We elected not to add other postAGB stars discussed by Maas (2003). Although these omissions are spectroscopic binaries and some show effects of dust-gas separation, available photometry does not show RV Tauri-like light variations. The run of $[\mathrm{Zn} / \mathrm{Fe}]$ for unevolved stars corresponds to $[\mathrm{Zn} / \mathrm{Fe}] \simeq 0$ for the range of $[\mathrm{Fe} / \mathrm{H}]$ covered in Figure 9 . Thickdisk stars may have $[\mathrm{Zn} / \mathrm{Fe}] \simeq+0.1$.

The limit $[\mathrm{Zn} / \mathrm{Fe}] \simeq 0.0$ represents the lower boundary to the distribution of the observed points. (The star with a distinctly negative value of $[\mathrm{Zn} / \mathrm{Fe}]$ is DF Cyg with a crowded spectrum and an uncertain $\mathrm{Zn}$ abundance.) Stars along the lower boundary are those with an abundance pattern indicating little or no dustgas separation. Several but not all high-velocity stars are near the boundary. The upper boundary to the data points follows the dashed line. This corresponds to approximately $[\mathrm{Zn} / \mathrm{Fe}]=$ $-[\mathrm{Fe} / \mathrm{H}]$, i.e., the trajectory of a star with an initial abundance $[\mathrm{Fe} / \mathrm{H}]=0$, which then became depleted in Fe to varying degrees while $\mathrm{Zn}$ remained undepleted. On the assumptions that $\mathrm{Fe}$, but not $\mathrm{Zn}$, is depleted and that initially $[\mathrm{Zn} / \mathrm{Fe}]=0$, the observed $\mathrm{Zn}$ abundance provides the star's initial Fe abundance $[\mathrm{Fe} / \mathrm{H}]_{0}$. By inspection, we see from Figure 9 that the $[\mathrm{Fe} / \mathrm{H}]_{0}$ runs from about +0.1 to -1.4 . This is shown by horizontal dotted line, corresponding to $[\mathrm{Zn} / \mathrm{Fe}] \sim 0$. It is shown by the figure that the dust-gas separation is not an either-or effect.

The run of $[\mathrm{S} / \mathrm{Fe}]$ versus $[\mathrm{Fe} / \mathrm{H}]$ (Fig. 10) resembles that in Figure 9 but with differences concerning the upper and lower boundaries. The lower boundary to the distribution of points runs systematically about 0.2 dex above the run of $[\mathrm{S} / \mathrm{Fe}]$ with $[\mathrm{Fe} / \mathrm{H}]$ for unevolved stars taken from Nissen et al. (2004). This offset is possibly attributable to systematic errors in one or both analyses. The dashed line corresponds to $[\mathrm{S} / \mathrm{Fe}]=[\mathrm{Fe} / \mathrm{H}]$. Several stars

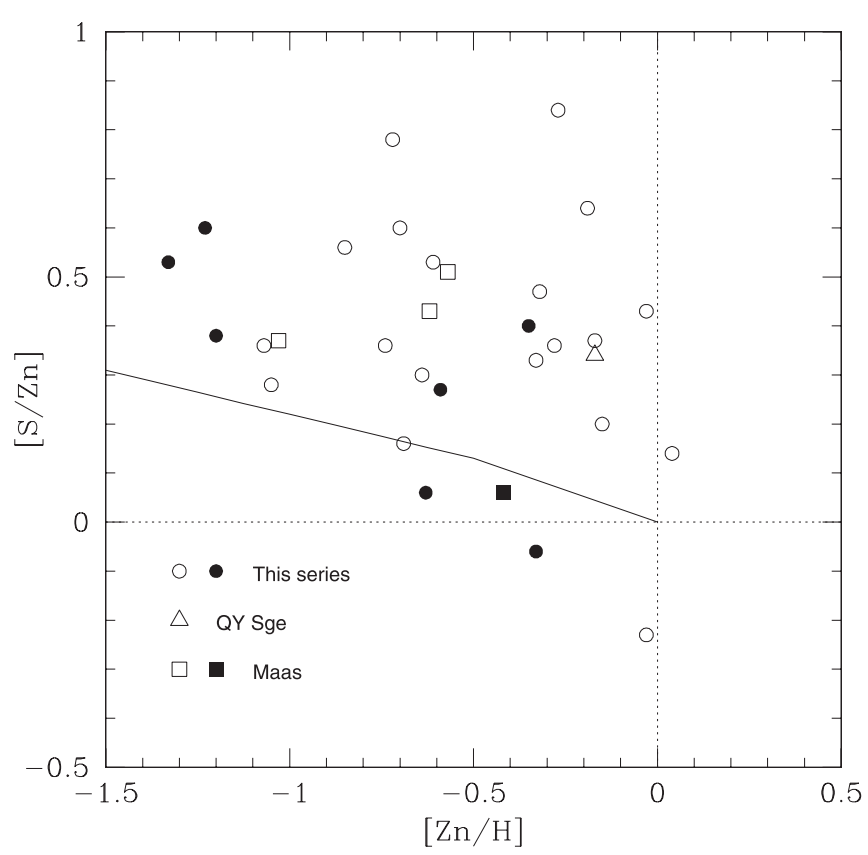

FIG. 11.-Abundance ratio $[\mathrm{S} / \mathrm{Zn}]$ vs. abundance $[\mathrm{Zn} / \mathrm{H}]$ for RV Tauri and related variables. RV Tauri stars from our series of papers are represented by circles. QY Sge (Kameswara Rao et al. 2002) is shown by the triangle. RV Tauri stars analyzed by Maas et al. (2002) and Maas (2003) are shown by squares. Filled circles and squares denote high-velocity stars. The solid line shows the run of $[\mathrm{S} / \mathrm{Zn}]$ with $[\mathrm{Zn} / \mathrm{H}]$ for unevolved stars (see text).

fall above this line. On the assumption that the $\mathrm{S}$ abundance is unaffected by dust-gas separation, the upper boundary in Figure 10 implies that the initial iron abundance for some stars is about $[\mathrm{Fe} / \mathrm{H}]_{0}=+0.4$, which seems implausible. A possible alternative explanation is that the $\mathrm{S}$ abundances for the RV Tauri stars are overestimated by about $0.2-0.4$ dex. These differences between Figures 9 and 10 necessarily make an appearance in Figure 11, which shows that the $[\mathrm{S} / \mathrm{Zn}]$ values of the RV Tauri stars fall generally above the expected trend for unevolved stars taken from Nissen et al.

In summary, we suggest that the initial $[\mathrm{Fe} / \mathrm{H}]$ of the RV Tauri and related stars is obtainable from their measured $\mathrm{Zn}$ abundance and the assumption that $[\mathrm{Zn} / \mathrm{Fe}]=0$ for normal stars.

\subsection{Carbon and Oxygen Abundances}

Main-sequence stars in evolving to red giants develop a deep convective envelope, which brings $\mathrm{CN}$-cycled products to the surface and thus reduces the surface $\mathrm{C}$ abundance and increases the surface $\mathrm{N}$ abundance (Iben 1967). Oxygen is expected to be very little affected. The reduction in the $\mathrm{C}$ abundance is $0.2-$ 0.3 dex (Lambert \& Ries 1981). After completion of He-core burning, material from the He-burning shell is dredged to the surface to increase the surface $\mathrm{C}$ abundance and also the $\mathrm{O}$ abundance. A star at this stage is known as an asymptotic giant branch (AGB) star. The He-burning shell may experience ignition of a neutron source and synthesis of heavy elements by the neutroncapture $s$-process. By examining the $\mathrm{C}, \mathrm{N}, \mathrm{O}$, and heavy element (e.g., Y and $\mathrm{Ba}$ ) abundances of the RV Tauri stars and taking due note of the initial abundances and the possible depletion of the surface abundances of these elements, one hopes to specify the evolutionary state of the RV Tauri variables.

In the following discussion, $[\mathrm{Zn} / \mathrm{H}]$ replaces $[\mathrm{Fe} / \mathrm{H}]$ as the representative of a star's initial composition. The initial $\mathrm{C}$ and $\mathrm{O}$ abundances as a function of $\mathrm{Zn}$ abundance are taken from measurements of unevolved stars (Nissen et al. 2004; Akerman 

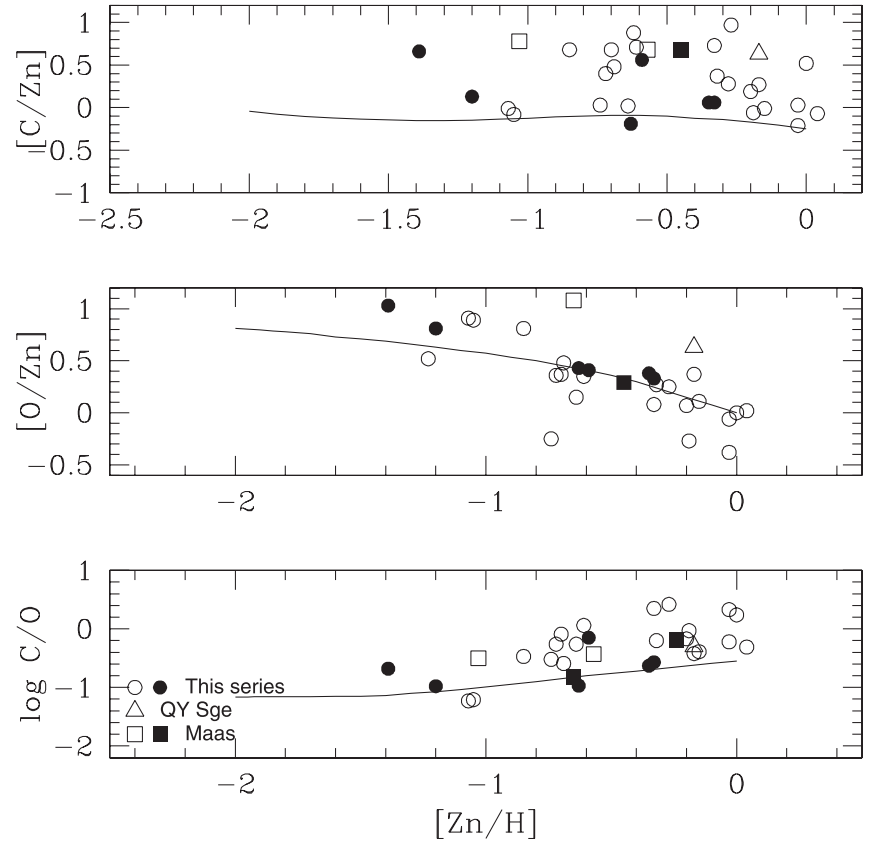

Fig. 12.-Abundances $[\mathrm{C} / \mathrm{Zn}]$ (top), $[\mathrm{O} / \mathrm{Zn}]$ (middle), and $\log \mathrm{C} / \mathrm{O}$ (bottom) as a function of the zinc abundance $[\mathrm{Zn} / \mathrm{H}]$ for $\mathrm{RV}$ Tauri and related variables. RV Tauri stars from our series of papers are represented by circles. QY Sge (Kameswara Rao et al. 2002) is shown by the triangle. Stars analyzed by Maas et al. (2002) and Maas (2003) are shown by squares. Filled circles and squares denote high-velocity stars. The solid line shows the expected runs with $[\mathrm{Zn} / \mathrm{H}]$ for giants after the first dredge-up (see text).

et al. 2004). We adopt the LTE abundances given by Akerman et al. (2004). Their tabulated NLTE abundances for oxygen are about $0.1-0.2$ dex smaller than the LTE values. NLTE abundances were not given for carbon. We assume that the first dredge-up experienced by a red giant reduced the initial $\mathrm{C}$ abundance by 0.25 dex but left the initial $\mathrm{O}$ abundance undisturbed.

The observed $\mathrm{C}$ abundances (Fig. 12) lie above the predictions for a red giant by about 0.4 dex, with a real star-to-star scatter. Carbon enrichment is suggested. However, a similar displacement of observed from initial abundances occurs for $[\mathrm{S} / \mathrm{Zn}]$. Since both the $\mathrm{C}$ and the $\mathrm{S}$ abundances are based on high-excitation atomic lines, the displacements may arise from similar systematic errors. The observed $\mathrm{O}$ abundances (Fig. 12) follow quite closely the trend of the initial abundances with $[\mathrm{Zn} / \mathrm{H}]$. The observed $\mathrm{C} / \mathrm{O}$ abundance ratios versus $[\mathrm{Zn} / \mathrm{H}]$ are shown in Figure 12 . The lower boundary to the observed ratios follows the predicted trend for first dredge-up red giants. These may be stars that suffered a reduction of surface $\mathrm{C}$ in becoming a red giant but did not later experience replenishment of $\mathrm{C}$. Stars with the highest $\mathrm{C} / \mathrm{O}$ ratio at a fixed $[\mathrm{Zn} / \mathrm{H}]$ fall about 0.5 dex above the predicted trend. This spread probably represents a real star-to-star spread in $\mathrm{C} / \mathrm{O}$. Very few stars are $\mathrm{C}$-rich, i.e., $\log \mathrm{C} / \mathrm{O} \geq 0$.

In summary, the RV Tauri variables have suffered differing degrees of surface enrichment of carbon. Some stars, especially stars of high velocity, have the $\mathrm{C} / \mathrm{O}$ ratio anticipated for fresh red giants; i.e., no obvious $\mathrm{C}$ enrichment occurred following the first dredge-up. Other stars have a $\mathrm{C} / \mathrm{O}$ ratio indicating up to a factor of 10 enrichment over the ratio of a fresh giant. Few stars are carbon-rich. There is no clear distinction in Figure 12 between the stars greatly affected by dust-gas separation and those unaffected.

\subsection{The s-Process Abundances}

Mature AGB stars are carbon-rich $(\mathrm{C} / \mathrm{O}>1)$ with enrichments of $s$-process products. Cool carbon stars on the AGB are $s$-process enriched by up to 1 dex (Abia et al. 2002). Post-AGB carbon-rich stars are similarly enriched (Reddy et al. 2002; Van Winckel 2003).

In the case of the RV Tauri stars unaffected by dust-gas separation, $s$-process abundances are directly obtainable from the measured abundances with respect to the iron abundance. No obvious enrichments are detected. For stars affected by dust-gas separation, the degree of $s$-process enrichment must be judged relative to the abundances of other elements of a similarly high $T_{C}$. Inspection of Figures 2-7 shows that there is no detectable overabundance of $s$-process elements. This is true for all other RV Tauri variables from our series of papers and those analyzed by Maas and colleagues. Our earlier suggestion that Ba may be overabundant in AR Pup and DS Aqr deserves reexamination. Barium, the only s-process element examined in AR Pup, was represented by a single strong line (Paper II). A similar situation applies to DS Aqr from Paper IV. New spectra of broad wavelength coverage should be obtained and analyzed before DS Aqr and AR Pup are tagged as " $s$-process-enriched."

In summary, the RV Tauri variables are unlikely to be descendants of luminous AGB stars in which s-process products from the He-burning shell have been dredged to the stellar surface.

\subsection{Boundary Conditions for Dust-Gas Separation}

Dust-gas separation is not ubiquitous among RV Tauri stars. Inspection of the stars unaffected by the separation shows that their temperature and metallicity domains are both well bounded. These bounds are surely clues to the site of the dust-gas separation and the process by which a star of anomalous surface composition is created.

Our sample of RV Tauri variables shows a low- and hightemperature boundary to the stars of anomalous composition. Stars of all metallicities with $T_{\text {eff }} \approx 5000 \mathrm{~K}$ and cooler are unaffected by dust-gas separation. ${ }^{4}$ This is shown by Figure 13. Our $T_{\text {eff }}$ estimates are spectroscopic determinations from spectra at phases showing well-defined symmetric absorption lines. The spectroscopic $T_{\text {eff }}$ may differ with phases (at which spectroscopic analysis is pursued) for a given star; for example, our three spectra of SS Gem gave $T_{\text {eff }}$ estimates of 4750,5500 , and $6500 \mathrm{~K}$ (Paper III). Effects of dust-gas separation are seen at the hightemperature end of the RV Tauri sample. This boundary is presumably set by the blue edge of the instability strip. Depletion is seen in hotter stars (Van Winckel 2003), notably the extremely metal-poor star HR 4049 with $T_{\text {eff }}=7600 \mathrm{~K}$ and with an observed iron abundance $[\mathrm{Fe} / \mathrm{H}] \simeq-4.8$. The oxygen abundance suggests an initial $[\mathrm{Fe} / \mathrm{H}]$ near -0.5 for HR 4049 .

In Paper V, we suggested that the coolest stars were unaffected because the convective envelope was of sufficient mass to dilute accreted gas even were it cleansed of dust. Frankowski (2003) discusses the mass of the convective envelope $\left(M_{\mathrm{CE}}\right)$ of postAGB stars by drawing on published numerical calculations. It is clear that $M_{\mathrm{CE}}$ increases with decreasing effective temperature: for example, $M_{\mathrm{CE}}$ at 4000,5000 , and $6000 \mathrm{~K}$ is $0.016,0.002$, and $0.001 M_{\odot}$, respectively. The $M_{\mathrm{CE}}$ at $6000 \mathrm{~K}$ is some 1000 times the mass of the observable photosphere. Abundance deficiencies for the high $T_{C}$ elements for stars on the hot side of the boundary may approach a thousandfold, and in such cases the surface and convective envelope must be composed of almost undiluted accreted gas. Although the increase of $M_{\mathrm{CE}}$ at lower temperatures

\footnotetext{
${ }^{4}$ Among the stars with $T_{\text {eff }} \leq 5000 \mathrm{~K}$, DY Aql appears to defy the above condition. Our analysis (Paper III) of DY Aql was based on very few lines; evidence of depletion rests almost exclusively on a lone Sc II line.
} 


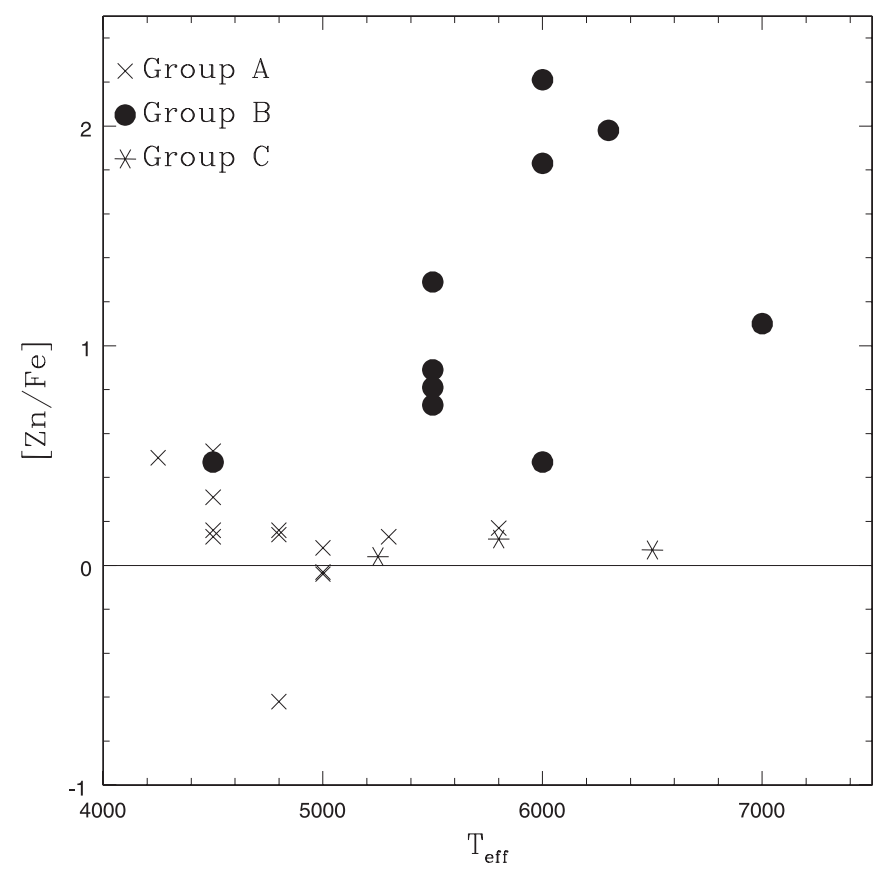

FIG. 13. - Abundance ratio $[\mathrm{Zn} / \mathrm{Fe}]$ vs. $T_{\text {eff }}$ for RV Tauri stars. Data points include the present work as well as data from our earlier papers. The ratio $[\mathrm{Zn} / \mathrm{Fe}]$ increases toward hotter temperature, indicating increasing severity of the dust-gas separation in hotter stars to cooler ones. Symbols represent Preston spectroscopic groups A, B, and C.

may play a role in suppressing the abundance anomalies, it hardly seems to offer the full explanation. Perhaps cooler variables have stronger winds, which impede accretion of gas from a circumbinary disk.

The dust-gas separation appears suppressed among stars of a low initial metallicity. Our sample of RV Tauri stars suggests that the limiting initial metallicity is $[\mathrm{Fe} / \mathrm{H}] \approx-1$. More metal-rich stars are affected, but less metal-rich stars do not show the abundance anomalies resulting from dust-gas separation, even if they are hotter than the above $T_{\text {eff }}$ boundary. For stars with $[\mathrm{Fe} / \mathrm{H}] \gtrsim$ -1 , there is no strong evidence that the dust-gas separation efficiency is sensitive to the initial metallicity.

In several examples, an affected RV Tauri star currently has a metallicity well below the limit $[\mathrm{Fe} / \mathrm{H}]$ of -1 for occurrence of a dust-gas separation. This is especially true for hotter stars such as HR 4049. Our inference from these observations is that it is the initial and not the current metallicity that is the key factor in establishing dust-gas separation. Hence, the dust-gas reservoir is a region with a metallicity in excess of $[\mathrm{Fe} / \mathrm{H}]$ of -1 . In a reservoir of low metallicity, separation of dust and gas is inhibited, and then if material is accreted from the reservoir by the star, the surface composition is unchanged.

There is a third boundary condition that may exist: effective dust-gas separation and accretion of gas but not dust by a star occurs efficiently only in a binary system. This is spectacularly the case for the small sample of hot post-AGB stars such as HR 4049 for which radial velocity monitoring has shown them to be single-lined spectroscopic binaries (Van Winckel et al. 1995). For the RV Tauri variables, detection of orbital variations in the face of pulsational variations is a complexity calling for long campaigns of radial velocity monitoring. Nonetheless, the number of RV Tauri variables known to be spectroscopic binaries is growing, as can be seen from Mass (2003). Van Winckel et al. (1998) have adduced indirect evidence in support of the idea that RV Tauri variables are binaries with a circumbinary disk.
Finally, there is the caution suggested by Kameswara Rao \& Reddy (2005) from their consideration of the compositions of EQ Cas and CE Vir, which showed that dust-gas separation may not always be the dominant factor behind abundance anomalies. We mentioned the special abundance anomalies of EQ Cas in $\S$ 3.3. We remarked on the lack of the usual correlation between $[\mathrm{X} / \mathrm{H}]$ and $T_{C}$. Kameswara Rao \& Reddy find that our $[\mathrm{X} / \mathrm{H}]-$ values for EQ Cas are anticorrelated with first ionization potential (FIP) of the elements. These authors suggest that the stellar wind preferentially picks up ions over neutral atoms. At the top of the stellar atmosphere, low FIP elements will be present as the ions $X^{+}$, but atoms of the high-FIP elements will remain neutral. The FIP effect is a minor contributor to the stars for which anomalies exhibit a strong $T_{C}$ correlation (Paper IV).

\section{CONCLUDING REMARKS}

Conversion of the boundary conditions into an understanding of how RV Tauri stars with and without abundance anomalies arise remains elusive. Two contrasting scenarios were sketched in Paper V: the stars with anomalous abundances are (1) single stars with dust-gas separation occurring in the stellar wind (the $\mathrm{S}$ hypothesis) and/or (2) binary stars with dust-gas separation occurring in a circumbinary disk (the $\mathrm{B}$ hypothesis). The boundary condition on $T_{\text {eff }}$ is met by both hypotheses if the deeper convective envelope for cooler stars acts to dilute the accreted gas. The boundary condition on initial metallicity seems more readily met by the $\mathrm{B}$ hypothesis; the circumbinary disk is comprised of gas ejected by either the RV Tauri's companion and/or the RV Tauri star before the onset of accretion and alteration of the photospheric abundances. Indeed, mass loss induced by a companion may not only provide the circumbinary gaseous disk but also shift the primary star off the giant branch and into the instability strip. This boundary condition is not easily satisfied for all stars by the S hypothesis; some RV Tauri stars have metal abundances less than the boundary $[\mathrm{Fe} / \mathrm{H}] \simeq-1$.

The B hypothesis provides a natural link between the RV Tauri stars and A-type, extremely metal-deficient stars such as HR 4049, which are known to be spectroscopic binaries. Binary RV Tauri stars are known. A determined campaign of radial velocity measurements for RV Tauri stars, especially those displaying anomalous abundances, is needed to establish the frequency of binaries. It should be noted, too, that the sample of HR 4049like stars is presently very small. A search for additional examples and a demonstration that they are (or are not!) spectroscopic binaries would also be valuable.

The B hypothesis requires gas to be accreted from the circumbinary disk by the star. This accretion, one presumes, competes with the wind from an RV Tauri star. Direct detection of the infalling gas should be sought. On the $\mathrm{S}$ hypothesis, it would seem that there must be a circulation of gas up to the dust-gas separation sites and back down to the photosphere. Spectroscopic evidence for this circulation may be obtainable from high-resolution spectroscopy over the pulsational period of a star with anomalous abundances.

Finally, we note that the sparsely populated region of the HR diagram that is home to RV Tauri variables and other luminous post-AGB stars deserves continued exploration. Van Winckel's (2003) review gives a thorough description and discussion of the compositions of post-AGB stars, of which few have "normal" abundances. Our study of RV Tauri stars began with a chance observation of IW Car. Perhaps continued but systematic spectroscopic exploration of post-AGB stars will uncover objects that will reveal vital clues to the operation of dust-gas separation in the RV Tauri and other stars. Galactic post-AGB stars offer the best opportunity for detailed study of the stellar photosphere and 
the circumstellar environment. Although considerably fainter, post-AGB stars in the Magellanic Clouds offer some advantages; e.g., their luminosities are obtainable with fair precision.
We thank the referee for a most thorough and constructive review of the paper. This research has been supported in part by the Robert A. Welch Foundation of Houston, Texas.
Abia, C., et al. 2002, ApJ, 579, 817

Akerman, C. J., Carigi, L., Nissen, P. E., Pettini, M., \& Asplund, M. 2004 A\&A, 414, 931

Busso, M., Gallino, R., \& Wasserburg, G. J. 1999, ARA\&A, 37, 239

Frankowski, A. 2003, A\&A, 406, 265

Giridhar, S., Kameswara Rao, N., \& Lambert, D. L. 1992, J. Astrophys. Astron., 13,307 1994, ApJ, 437, 476 (Paper I)

Giridhar, S., Lambert, D. L., \& Gonzalez, G. 1998, ApJ, 509, 366 (Paper IV) 2000, ApJ, 531, 521 (Paper V)

Gonzalez, G., \& Lambert, D. L. 1997, AJ, 114, 341

Gonzalez, G., Lambert, D. L., \& Giridhar, S. 1997a, ApJ, 479, 427 (Paper II) 1997b, ApJ, 481, 452 (Paper III)

Goswami, A., \& Prantzos, N. 2000, A\&A, 359, 191

Graczyk, D., Mikołajewski, M., Leedjärv, L., Frąckowiak, S. M., Osiwała, J. P.,

Puss, A., \& Tomov, T. 2002, Acta Astron., 52, 293

Iben, I., Jr. 1967, ARA\&A, 5, 571

Kameswara Rao, N., Goswami, A., \& Lambert, D. L. 2002, MNRAS, 334, 129

Kameswara Rao, N., \& Reddy, B. E. 2005, MNRAS, 357, 235

Kholopov, P. N., Samus, N. N., Durlevich, O. V., Kazarovets, E. V., Kireeva,

N. N., \& Tsvetkova, T. M. 1992, General Catalogue of Variable Stars (4th ed.; Moscow: Nauka)

Kukarkin, B. V., Parenago, P. P., Yu, N., \& Kholopov, P. N. 1958, General Catalogue of Variable Stars (2nd ed.; Moscow: Acad. Sci. USSR)

Kurucz, R. L. 1993, CD-ROM 13, ATLAS9 Stellar Atmosphere Program and $2 \mathrm{~km} / \mathrm{s}$ Grid (Cambridge: SAO)

Lambert, D. L., \& Ries, L. M. 1981, ApJ, 248, 228
REFERENCES

Lloyd Evans, T. 1999, in IAU Symp. 191, Asymptotic Giant Branch Stars, ed. T. Le Bertre, A. Lèbre, \& C. Waelkens (San Francisco: ASP), 453

Lodders, K. 2003, ApJ, 591, 1220

Lodders, K., \& Fegley, B. 1998, The Planetary Scientist's Companion (New York: Oxford Univ. Press)

Luck, R. E. 1981, PASP, 93, 211

Maas, T. 2003, Ph.D. thesis, Univ. Leuven

Maas, T., Van Winckel, H., \& Waelkens, C. 2002, A\&A, 386, 504

McCarthy, J. K., Sandiford, B. A., Boyd, D., \& Booth, J. 1993, PASP, 105, 88

Mishenina, T. V., Kovtyukh, V. V., Soubiran, C., Travaglio, C., \& Busso, M. 2002, A\&A, 396, 189

Nissen, P. E., Chen, Y. Q., Asplund, M., \& Pettini, M. 2004, A\&A, 415, 993

Preston, G. W., Krzeminski, W., Smak, J., \& Williams, J. A. 1963, ApJ, 137, 401

Prochaska, J. X., Naumov, S. O., Carney, B. W., McWilliam, A., \& Wolfe, A. M. 2000, AJ, 120, 2513

Reddy, B. E., Lambert, D. L., Gonzalez, G., \& Yong, D. 2002, ApJ, 564, 482

Reddy, B. E., Tomkin, J., Lambert, D. L., \& Allende Prieto, C. 2003, MNRAS, 340,304

Russell, S. C. 1998, Publ. Astron. Soc. Australia, 15, 189

Ryde, N., \& Lambert, D. L. 2004, A\&A, 415, 559

Savage, B. D., \& Sembach, K. R. 1996, ARA\&A, 34, 279

Sneden, C. 1973, Ph.D. thesis, Univ. Texas, Austin

Tull, R. G., MacQueen, P. J., Sneden, C., \& Lambert, D. L. 1995, PASP, 107, 251

Van Winckel, H. 2003, ARA\&A, 41, 391

Van Winckel, H., Waelkens, C., \& Waters L. B. F. M. 1995, A\&A, 293, L25

Van Winckel, H., Waelkens, C., Waters, L. B. F. M., Molster, F. J., Udry, S., \& Bakker, E. J. 1998, A\&A, 336, L17 